\title{
On-shell constructibility of Born amplitudes in spontaneously broken gauge theories
}

\author{
Robert Franken $^{a}$ and Christian Schwinn ${ }^{b}$ \\ ${ }^{a}$ Institut für Theoretische Physik und Astrophysik, Universität Würzburg, \\ D-97074 Würzburg, Germany \\ ${ }^{b}$ Institut für Theoretische Teilchenphysik und Kosmologie, RWTH Aachen University, \\ D-52056 Aachen, Germany \\ E-mail: robert.franken@physik.uni-wuerzburg.de, \\ schwinn@physik.rwth-aachen.de
}

ABSTRACT: We perform a comprehensive study of on-shell recursion relations for Born amplitudes in spontaneously broken gauge theories and identify the minimal shifts required to construct amplitudes with a given particle content and spin quantum numbers. We show that two-line or three-line shifts are sufficient to construct all amplitudes with five or more particles, apart from amplitudes involving longitudinal vector bosons or scalars, which may require at most five-line shifts. As an application, we revisit selection rules for multi-boson amplitudes using on-shell recursion and little-group transformations.

KEywords: Scattering Amplitudes, Spontaneous Symmetry Breaking

ARXIV EPRINT: 1910.13407 


\section{Contents}

1 Introduction 1

2 Spinor formalism for massive particles $\quad 2$

2.1 Spinor variables for massive momenta 2

2.2 Little-group transformations 4

2.3 Massive fermions 5

2.4 Massive vector bosons 6

3 Complex continuation of massive amplitudes $\quad 7$

$\begin{array}{lr}3.1 \text { Shifts of massive momenta } & 9\end{array}$

$\begin{array}{lll}3.2 & \text { Shifts of wave-functions } & 11\end{array}$

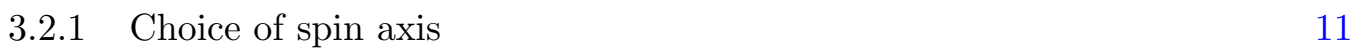

$\begin{array}{lll}3.3 & \text { Multi-line shifts } & 12\end{array}$

$\begin{array}{lll}\text { 3.3.1 Two-line BCFW shifts } & 14\end{array}$

$\begin{array}{lll}\text { 3.3.2 Multi-line Risager-type shifts } & 14\end{array}$

$\begin{array}{lll}\text { 3.3.3 Multi-line BCFW-type shifts } & 15\end{array}$

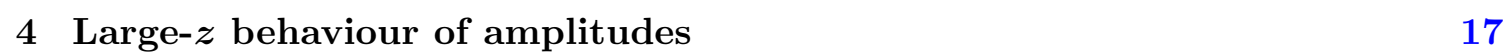

4.1 Scaling of external states and Goldstone-boson equivalence 18

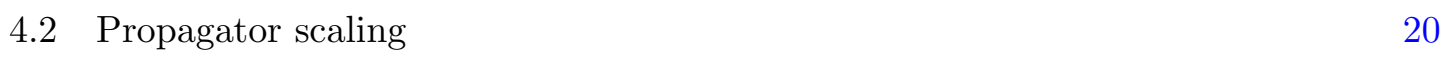

$\begin{array}{lll}4.3 & \text { Bounds for generic shifts with } Q_{\mathcal{F}}^{2}=0 & 20\end{array}$

4.4 Bounds for multi-line Risager and BCFW shifts 21

$\begin{array}{lll}\text { 4.4.1 Multi-line Risager-type shifts } & 22\end{array}$

4.4.2 Multi-line BCFW-type shifts 23

4.5 Examples 25

5 On-shell constructible amplitudes $\quad 27$

5.1 Choice of reference spinors for all spin configurations 28

$\begin{array}{ll}5.2 \text { Explicit form of shifts } & 29\end{array}$

$\begin{array}{lll}5.3 & \text { Minimal required shifts } & 30\end{array}$

$\begin{array}{lll}\text { 5.3.1 } & \text { Five-line constructible amplitudes } & 31\end{array}$

$\begin{array}{lll}\text { 5.3.2 } & \text { Four-line constructible amplitudes } & 31\end{array}$

$\begin{array}{lll}\text { 5.3.3 Three-line constructible amplitudes } & 31\end{array}$

6 Application to selection rules $\quad 33$

6.1 Selection rules for bosonic amplitudes 34

6.2 Selection rules for amplitudes with a fermion pair 36

$\begin{array}{ll}\text { 6.3 Transformation to a common spin axis } & 38\end{array}$

$\begin{array}{lll}7 & \text { Summary and conclusions } & 40\end{array}$ 


\section{Introduction}

The development of on-shell recursion relations of Born amplitudes in gauge theories by Britto, Cachazo, Feng, and Witten (BCFW) [1, 2] has motivated an approach to Quantum Field Theory that aims at the construction of amplitudes solely in terms of on-shell building blocks. For massless theories, space-time symmetries and factorization properties are sufficient to fix the structure of three- and four-point amplitudes and to establish the uniqueness of non-abelian gauge theories [3]. The three-point vertices serve as input to the $\mathrm{BCFW}$ recursion relations, which are based on a continuation of amplitudes into the complex plane by a complex shift of two external momenta. This provides a purely on-shell construction of Born amplitudes in unbroken gauge theories. The on-shell constructibility of gauge theories with general matter content or of Effective Field Theories was investigated using generalizations of the BCFW construction to shifts of more than two legs [4-6].

Concerning theories with massive particles, symmetry constraints on three-point amplitudes were obtained using supersymmetry [7, 8] and little-group transformations $[9,10]$. More recently, this analysis was simplified using a manifestly little-group covariant notation for massive amplitudes [11]. The uniqueness of four-point amplitudes can be argued to arise from factorization properties and consistency with the high-energy limit [11]. This line of argument is expected to reproduce the classic results on the uniqueness of spontaneously broken gauge theories (SBGTs) [12-14] within an on-shell approach. All three-point vertices and amplitudes for some three- and four-body decays in the electroweak Standard Model (SM) were constructed using this formalism [15, 16], while all three-point vertices including contributions from higher-dimensional operators were obtained in [17].

In the current paper we investigate the on-shell constructibility of higher-point Born amplitudes in SBGTs. After initial explorations of BCFW recursion for amplitudes with massive particles [18-22], it was shown that amplitudes in QCD with massive quarks can be constructed using at most three-line shifts [23] whereas the on-shell constructibility of amplitudes in massive, power-counting renormalizable theories using all-line shifts was proven in [4]. The constructibility of massless amplitudes with the matter content of SBGTs using at most five-line shifts was demonstrated in [5]. We extend this analysis to the broken phase and show that all amplitudes involving at least two transverse vector bosons can be constructed using three-line shifts, while amplitudes with longitudinal vector bosons or scalars may require four- or five-line shifts. This requires the analysis of the behaviour of amplitudes for large values of the parameter $z$, which parameterises the complex continuation of the amplitude. Because of the analogy of the large- $z$ limit with a high-energy 
limit [24], it is anticipated that the result of [5] carries over to the broken phase. However, in the analysis of massive amplitudes we encounter several technical complications compared to the massless case. In particular, obtaining shifts with a "good" large- $z$ behaviour appears to make it necessary to break manifest little-group covariance by introducing a fixed spin axis. This requires a careful analysis to ensure that amplitudes for arbitrary spin configurations can be constructed recursively. Furthermore the violation of helicity selection rules by mass terms can lead to contributions to amplitudes with a worse large- $z$ behaviour than in the massless case.

The paper is structured as follows. In section 2 the little-group covariant spinor formalism for massive particles [11] is reviewed and related to expressions for Dirac spinors and polarization vectors for a fixed spin axis. In section 3 a systematic discussion of shifts of massive momenta is given, generalizing the extended Risager [25] and BCFW-type shifts used in [5] to the massive case. After initially constructing shifts of momenta and wavefunctions in a little-group covariant form, a suitable choice of spin axes leads to a similar $z$-dependence of wave functions as in the massless case. All the required shifts with two to five legs are constructed explicitly. The large- $z$ behaviour of amplitudes in SBGTs is established in section 4 . These results are used in section 5 to identify the minimal number of shifted lines needed to construct a given amplitude. As an application, in section 6 selection rules for amplitudes with massive vector bosons are derived using recursion relations and little-group transformations, providing a new perspective on results obtained using diagrammatic analysis [26] or supersymmetry [8]. Details on the employed spinor conventions and explicit expressions for little-group transformations changing the spin axis are given in appendices A and B, respectively. Recursion relations with non-lightlike shifts of internal lines are briefly discussed in appendix C.

\section{Spinor formalism for massive particles}

In this section the conventions for the momenta and wave-functions of massive particles are set up, relating the little-group covariant notation introduced recently in [11] to the conventions used previously for on-shell recursion relations for massive quarks [23]. The use of little-group transformations to relate wave-functions with different spin quantum numbers is also discussed.

\subsection{Spinor variables for massive momenta}

At the heart of the spinor-helicity method is the observation that the complex two-by-two matrix $k_{\alpha \dot{\alpha}}=k_{\mu} \sigma_{\alpha \dot{\alpha}}^{\mu}$ associated to a light-like momentum factorizes into a product of twocomponent Weyl spinors in the two inequivalent fundamental representations of $\operatorname{SL}(2, \mathbb{C})$,

$$
k_{\alpha \dot{\alpha}}=k_{\alpha} k_{\dot{\alpha}}
$$

where we refer to the spinors $k_{\alpha} \in D^{\left(\frac{1}{2}, 0\right)}$ as holomorphic and the spinors in the conjugate representation $k_{\dot{\alpha}} \in D^{\left(0, \frac{1}{2}\right)}$ as anti-holomorphic. Our Weyl-spinor conventions follow [23] 
and are summarized in appendix A. Similarly, a massive momentum satisfying the onshell condition

$$
k_{\alpha \dot{\alpha}} k^{\dot{\alpha} \beta}=m^{2} \delta_{\alpha}^{\beta}
$$

can be expanded in a basis of two holomorphic and two anti-holomorphic spinors. Various approaches have been followed in the literature, e.g. introducing fixed spin vectors [27] or using helicity eigenstates [28].

A notation that makes the transformation properties of spinor variables under the little group $\mathrm{SU}(2)$ of massive momenta manifest was introduced in [11]. In this notation, a massive momentum is parameterized as

$$
k_{\alpha \dot{\alpha}}=k_{\alpha}^{I} k_{\dot{\alpha}, I}=k_{\alpha}^{I} k_{\dot{\alpha}}^{J} \varepsilon_{J I}, \quad \quad k^{\dot{\alpha} \alpha}=k_{I}^{\dot{\alpha}} k^{\alpha, I}=k_{I}^{\dot{\alpha}} k_{J}^{\alpha} \varepsilon^{I J},
$$

where the two-component little-group indices $I$ are raised and lowered with the twodimensional antisymmetric tensor. Using the same conventions as for un-dotted Weylspinor indices in (A.3), these relations read

$$
k_{\alpha}^{I}=\varepsilon^{I J} k_{\alpha, J}, \quad k_{\alpha, J}=k_{\alpha}^{I} \varepsilon_{I J}, \quad \varepsilon^{I J}=\varepsilon_{I J}=\left(\begin{array}{cc}
0 & 1 \\
-1 & 0
\end{array}\right),
$$

with identical definitions for the anti-holomorphic spinors.

The massive spinor variables can be chosen to satisfy the $\mathrm{SU}(2)$ and $\mathrm{SL}(2, \mathbb{C})$ covariant normalization conventions

$$
\begin{aligned}
\left\langle k^{I} k^{J}\right\rangle & =m \varepsilon^{I J}, & {\left[k_{I} k_{J}\right] } & =-m \varepsilon_{I J}, \\
k_{\alpha}^{I} k_{\beta, I} & =m \varepsilon_{\alpha \beta}, & k_{\dot{\alpha}}^{I} k_{\dot{\beta}, I} & =m \varepsilon_{\dot{\alpha} \dot{\beta}} .
\end{aligned}
$$

Holomorphic and anti-holomorphic spinors are related by the Dirac equations

$$
k_{\alpha \dot{\alpha}} k_{I}^{\dot{\alpha}}=-m k_{\alpha, I}, \quad \quad k^{\dot{\alpha} \alpha} k_{\alpha}^{I}=-m k^{\dot{\alpha}, I} .
$$

In the construction of the complex continuation of scattering amplitudes we will be forced to break manifest little-group covariance and fix a particular basis for the decomposition of the momenta, as in earlier work on on-shell recursion for massive momenta [23]. In this reference a fixed, light-like reference momentum $q$ is used to decompose a massive momentum into a sum of two light-like vectors,

$$
k^{\mu}=k^{b ; \mu}+\frac{m^{2}}{2(q \cdot k)} q^{\mu} .
$$

The associated holomorphic and anti-holomorphic Weyl spinors $k_{\alpha}^{b}, q_{\alpha}$ and $k_{\dot{\alpha}}^{b}, q_{\dot{\alpha}}$ provide a particular example of a basis for the expansion of a massive momentum, which corresponds to the choice

$$
\begin{aligned}
k_{\alpha}^{1}=k_{\alpha}^{b}, & k_{\alpha}^{2}=\frac{m}{\left\langle k^{b} q\right\rangle} q_{\alpha}, \\
k_{\dot{\alpha}, 1}=k_{\dot{\alpha}}^{b}, & k_{\dot{\alpha}, 2}=\frac{m}{\left[q k^{b}\right]} q_{\dot{\alpha}}
\end{aligned}
$$


in the little-group covariant expressions. External wave-functions of massive particles can be defined as eigenstates of the corresponding spin operators with respect to the spin vector

$$
n_{q}^{\mu}=\frac{k^{\mu}}{m}-\frac{m q^{\mu}}{q \cdot k}
$$

as discussed e.g. in [8].

\section{$2.2 \quad$ Little-group transformations}

By definition, little-group transformations $R \in \mathrm{SU}(2)$ of the Weyl spinors,

$$
k^{I} \rightarrow k^{I}=R_{J}^{I} k^{J}, \quad k_{I} \rightarrow k_{I}^{\prime}=-R_{I}^{J} k_{J},
$$

leave the momenta (2.3) invariant. These definitions hold both for dotted and undotted $\mathrm{SL}(2, \mathbb{C})$ indices, which have been suppressed. Note that spinors with lower little-group indices transform in the dual representation with the transformations

$$
\left(R^{T^{-1}}\right)_{I}^{J}=\left(\varepsilon^{-1}\right)_{I K} R^{K}{ }_{L} \varepsilon^{L J}=-R_{I}^{J},
$$

where indices of the little-group rotations are raised and lowered with the convention (2.4).

Infinitesimal little-group transformations,

$$
R_{J}^{I}=\delta_{J}^{I}+\omega^{I}+\ldots,
$$

are parameterized by three parameters in the symmetric matrix $\omega^{I J}=\omega^{J I}$. The action of infinitesimal little-group transformations on functions of the spinor variables of a momentum $k$ induces a representation of the Lie algebra of the little group,

$$
\delta \varphi\left(k_{\alpha}^{I}, k_{\dot{\alpha}}^{I}\right)=\varphi\left(k_{\alpha}^{\prime I}, k_{\dot{\alpha}}^{\prime I}\right)-\varphi\left(k_{\alpha}^{I}, k_{\dot{\alpha}}^{I}\right)=-\omega^{I}{ }_{J}\left(J_{k}\right)^{J}{ }_{I} \varphi+\mathcal{O}\left(\omega^{2}\right),
$$

with the differential operators $[9,10]$

$$
\left(J_{k}\right)^{J}{ }_{I}=-\left(k_{\alpha}^{J} \frac{\partial}{\partial k_{\alpha}^{I}}+k_{\dot{\alpha}}^{J} \frac{\partial}{\partial k_{\dot{\alpha}}^{I}}\right) .
$$

For the determination of spin eigenstates for a fixed spin axis it is useful to form the linear combinations

$$
\begin{aligned}
J_{k}^{-} & \equiv\left(J_{k}\right)^{1}{ }_{2}=\left(-\frac{\left\langle k^{b} q\right\rangle}{m} k_{\alpha}^{b} \frac{\partial}{\partial q_{\alpha}}+\frac{m}{\left[q k^{b}\right]} q_{\dot{\alpha}} \frac{\partial}{\partial k_{\dot{\alpha}}^{b}}\right), \\
J_{k}^{0} & \equiv \frac{1}{2}\left(\left(J_{k}\right)^{1}{ }_{1}-\left(J_{k}\right)^{2}{ }_{2}\right)=-\frac{1}{2}\left(k_{\alpha}^{b} \frac{\partial}{\partial k_{\alpha}^{b}}-q_{\alpha} \frac{\partial}{\partial q_{\alpha}}-k_{\dot{\alpha}}^{b} \frac{\partial}{\partial k_{\dot{\alpha}}^{b}}+q_{\dot{\alpha}} \frac{\partial}{\partial q_{\dot{\alpha}}}\right), \\
J_{k}^{+} & \equiv\left(J_{k}\right)^{2}{ }_{1}=\left(-\frac{m}{\left\langle k^{b} q\right\rangle} q_{\alpha} \frac{\partial}{\partial k_{\alpha}^{b}}+\frac{\left[q k^{b}\right]}{m} k_{\dot{\alpha}}^{b} \frac{\partial}{\partial q_{\dot{\alpha}}}\right) .
\end{aligned}
$$

These operators satisfy the commutation relations

$$
\left[J_{k}^{0}, J_{k}^{ \pm}\right]= \pm J_{k}^{ \pm}, \quad\left[J_{k}^{+}, J_{k}^{-}\right]=2 J_{k}^{0},
$$

which show that $J_{k}^{ \pm}$serve as raising and lowering operators for the eigenstates of $J_{k}^{0}$. 


\subsection{Massive fermions}

Solutions to the massive Dirac equation for particle spinors and their conjugates,

$$
(\not k-m) u(k)=0, \quad \bar{u}(k)(\not k-m)=0,
$$

can be constructed in the little-group covariant notation as

$$
u^{I}(k)=\left(\begin{array}{c}
k_{\alpha}^{I} \\
-k^{\dot{\alpha}, I}
\end{array}\right), \quad \bar{u}_{I}(k)=\left(k_{I}^{\alpha}, k_{\dot{\alpha}, I}\right) .
$$

Up to different sign conventions these expressions agree with those of [29] where it is shown that they form helicity eigenstates. They satisfy the conventional completeness and normalization conditions due to the properties of the two-component spinors (2.6):

$$
\begin{aligned}
& u^{I}(k) \bar{u}_{I}(k)=\left(\begin{array}{cc}
k_{\alpha}^{I} k_{I}^{\beta} & k_{\alpha}^{I} k_{\dot{\beta}, I} \\
-k^{\dot{\alpha}, I} k_{I}^{\beta} & -k^{\dot{\alpha}, I} k_{\dot{\beta}, I}
\end{array}\right)=\left(\begin{array}{cc}
m \delta_{\alpha}^{\beta} & k_{\alpha \dot{\beta}} \\
k^{\dot{\alpha} \beta} & m \delta_{\dot{\beta}}^{\dot{\alpha}}
\end{array}\right)=\not k+m, \\
& \bar{u}_{I}(k) u^{J}(k)=\left\langle k_{I} k^{J}\right\rangle-\left[k_{I} k^{J}\right]=2 m \delta_{I}^{J} .
\end{aligned}
$$

The little-group transformation of the Dirac spinors follows from the index positions,

$$
u^{I} \rightarrow R_{J}^{I} u^{J}, \quad \bar{u}_{I} \rightarrow-\bar{u}_{J} R_{I}^{J} .
$$

Using the translation (2.9) it is seen that the Dirac spinors (2.19) are related to expressions for a fixed spin axis in the conventions of [8] by the correspondence

$$
\begin{array}{ll}
u\left(k, \frac{1}{2}\right)=u^{2}(k)=\left(\begin{array}{cl}
\frac{m}{\left\langle k^{b} q\right\rangle} q_{\alpha} \\
k^{b ;}
\end{array}\right), & u\left(k,-\frac{1}{2}\right)=u^{1}(k)=\left(\begin{array}{c}
k_{\alpha}^{b} \\
-\frac{m}{\left[q k^{b}\right]} q^{\dot{\alpha}}
\end{array}\right), \\
\bar{u}\left(k, \frac{1}{2}\right)=\bar{u}_{1}(k)=\left(-\frac{m}{\left\langle k^{b} q\right\rangle} q^{\alpha}, k_{\dot{\alpha}}^{b}\right), & \bar{u}\left(k,-\frac{1}{2}\right)=\bar{u}_{2}(k)=\left(k^{b ; \alpha}, \frac{m}{\left[q k^{b}\right]} q_{\dot{\alpha}}\right) .
\end{array}
$$

Suitable expressions for the corresponding antiparticle spinors are given by

$$
v_{I}(k)=\left(\begin{array}{c}
k_{\alpha, I} \\
k_{I}^{\dot{\alpha}}
\end{array}\right), \quad \bar{v}^{I}(k)=\left(k^{\alpha, I},-k_{\dot{\alpha}}^{I}\right),
$$

which are identified with the expressions for a fixed spin axis as

$$
\begin{aligned}
& v\left(k, \frac{1}{2}\right)=v_{1}(k)=\left(\begin{array}{c}
-\frac{m}{\left\langle k^{b} q\right\rangle} q_{\alpha} \\
k^{b ; \dot{\alpha}}
\end{array}\right), \quad v\left(k,-\frac{1}{2}\right)=v_{2}(k)=\left(\begin{array}{c}
k_{\alpha}^{b} \\
\frac{m}{\left[q k^{b}\right]} q^{\dot{\alpha}}
\end{array}\right), \\
& \bar{v}\left(k, \frac{1}{2}\right)=\bar{v}^{2}(k)=\left(\frac{m}{\left\langle k^{b} q\right\rangle} q^{\alpha}, k_{\dot{\alpha}}^{b}\right), \quad \bar{v}\left(k,-\frac{1}{2}\right)=\bar{v}^{1}(k)=\left(k^{b ; \alpha},-\frac{m}{\left[q k^{b}\right]} q_{\dot{\alpha}}\right) .
\end{aligned}
$$

The spinors $\bar{u}(k, s)$ and $v(k, s)$ describe outgoing particles and antiparticles with spin quantum number $s$, while $u(k, s)$ and $\bar{v}(k, s)$ describe incoming particles and antiparticles with reversed spin label. For the Weyl-spinor conventions of appendix A the behaviour of spinors under a reversal of the momentum is given by

$$
v(-k, s)=\mathrm{i} \operatorname{sgn}\left(k^{0}+k^{3}\right) u(k, s), \quad \bar{v}(-k, s)=\mathrm{i} \operatorname{sgn}\left(k^{0}+k^{3}\right) \bar{u}(k, s) .
$$


A change of the spin axis corresponds to a little-group rotation of the Dirac spinors,

$$
u^{\prime}\left(s^{\prime}\right)=\sum_{s= \pm \frac{1}{2}} \mathcal{R}_{s^{\prime} s}^{\left(\frac{1}{2}\right)} u(s), \quad \quad \bar{u}^{\prime}\left(s^{\prime}\right)=\sum_{s= \pm \frac{1}{2}} \bar{u}(s) \mathcal{R}_{s s^{\prime}}^{\left(\frac{1}{2}\right)-1}
$$

where the matrix $\mathcal{R}^{\left(\frac{1}{2}\right)}$ is given explicitly in (B.4) in appendix B.

The Dirac spinors are eigenstates of the generators $J^{0}$,

$$
J_{k}^{0} u(k, s)=s u(k, s), \quad J_{k}^{0} v(k, s)=s v(k, s),
$$

and related by the action of $J^{ \pm}$in (2.16),

$$
\begin{array}{ll}
J_{k}^{ \pm} u\left(k, \pm \frac{1}{2}\right)=0, & J_{k}^{ \pm} u\left(k, \mp \frac{1}{2}\right)=-u\left(k, \pm \frac{1}{2}\right), \\
J_{k}^{ \pm} v\left(k, \pm \frac{1}{2}\right)=0, & J_{k}^{ \pm} v\left(k, \mp \frac{1}{2}\right)=v\left(k, \pm \frac{1}{2}\right),
\end{array}
$$

which are the expected relations for angular-momentum ladder operators up to a nonstandard phase convention for the particle spinors.

\subsection{Massive vector bosons}

Polarization vectors of massive spin one particles transform under the three-dimensional representation of the little group and can be described by symmetric bi-spinors,

$$
\epsilon_{\alpha \dot{\alpha}}^{(I J)}(k)=\frac{1}{\sqrt{2} m} k_{\alpha}^{(I} k_{\dot{\alpha}}^{J)}=\frac{1}{\sqrt{2} m}\left(k_{\alpha}^{I} k_{\dot{\alpha}}^{J}+k_{\alpha}^{J} k_{\dot{\alpha}}^{I}\right) \text {. }
$$

These satisfy the transversality, orthonormality, and completeness relations

$$
\begin{aligned}
k^{\dot{\alpha} \alpha} \epsilon_{\alpha \dot{\alpha}}^{(I J)} & =0, \\
\epsilon_{\alpha \dot{\alpha}}^{(I J)} \epsilon^{(K L) \dot{\alpha} \alpha} & =\left(\varepsilon^{I L} \varepsilon^{J K}+\varepsilon^{J L} \varepsilon^{I K}\right), \\
\epsilon_{\alpha \dot{\alpha}}^{(I J)} \epsilon_{(I J)}^{\dot{\beta} \beta} & =\left(\delta_{\alpha}^{\beta} \delta_{\dot{\alpha}}^{\dot{\beta}}-\frac{k_{\alpha}^{\dot{\beta}} k_{\dot{\alpha}}^{\beta}}{m^{2}}\right) .
\end{aligned}
$$

The polarization vectors transform under little-group transformations as second-rank tensors,

$$
\epsilon^{(I J)} \rightarrow R^{I}{ }_{M} R^{J} \epsilon^{(M N)} .
$$

The expressions in the spinor formalism for the fixed spin axis (see e.g. [8, 28]),

$$
\epsilon_{\alpha \dot{\alpha}}(k,+)=\sqrt{2} \frac{q_{\alpha} k_{\dot{\alpha}}^{b}}{\left\langle q k^{b}\right\rangle}, \quad \epsilon_{\alpha \dot{\alpha}}(k,-)=\sqrt{2} \frac{k_{\alpha}^{b} q_{\dot{\alpha}}}{\left[k^{b} q\right]}, \quad \epsilon_{\alpha \dot{\alpha}}(k, 0)=\frac{1}{m}\left(k_{\alpha}^{b} k_{\dot{\alpha}}^{b}-\frac{m^{2}}{2 q \cdot k} q_{\alpha} q_{\dot{\alpha}}\right),
$$

are related to the little-group covariant notation by the identifications

$$
\epsilon(k,+)=\epsilon^{(22)}(k), \quad \epsilon(k,-)=-\epsilon^{(11)}(k), \quad \epsilon(k, 0)=-\sqrt{2} \epsilon^{(12)}(k),
$$


where the minus sign in the definition of $\epsilon(k,-)$ ensures the conventional normalization condition

$$
\frac{1}{2} \epsilon_{\alpha \dot{\alpha}}(\lambda) \epsilon^{\dot{\alpha} \alpha}\left(-\lambda^{\prime}\right)=\epsilon(\lambda) \cdot \epsilon\left(-\lambda^{\prime}\right)=-\delta_{\lambda, \lambda^{\prime}} .
$$

As a result of these conventions, the action of the generators (2.16) on the polarization vectors is given by

$$
\begin{aligned}
J_{k}^{0} \epsilon(k, s) & =s \epsilon(k, s), \\
J_{k}^{ \pm} \epsilon(k, \pm) & =0, \quad J_{k}^{\mp} \epsilon(k, \pm)= \pm \sqrt{2} \epsilon(k, 0), \quad J_{k}^{ \pm} \epsilon(k, 0)= \pm \sqrt{2} \epsilon(k, \pm) .
\end{aligned}
$$

A change of the spin axis is represented as a little-group transformation,

$$
\epsilon^{\prime}\left(s^{\prime}\right)=\sum_{s=+, 0,-} \mathcal{R}_{s^{\prime} s}^{(1)} \epsilon(s),
$$

where the matrix $\mathcal{R}^{(1)}$ is given in (B.5).

\section{Complex continuation of massive amplitudes}

The basis of the proof [2] of the original on-shell recursion relation [1] and its generalizations $[4,5,25]$ is the construction of a complex continuation $A(z)$ of scattering amplitudes obtained by a deformation of a subset $\mathcal{S}=\left\{k_{i}\right\}, i=1, \ldots h$ of the four-momenta of the external particles, parameterized by a complex parameter $z$,

$$
k_{i} \rightarrow \hat{k}_{i}(z) \text { for } \quad k_{i} \in \mathcal{S}
$$

so that the physical amplitude is given by $A(0)$. We consider deformations with the following properties:

- Four-momenta are deformed by a linear shift in $z$,

$$
\hat{k}_{i}(z)=k_{i}+z \delta k_{i} .
$$

- The shift does not modify the mass-shell condition of the external momenta,

$$
\hat{k}_{i}^{2}(z)=k_{i}^{2}=m_{i}^{2} .
$$

- The deformed momenta satisfy momentum conservation if the original momenta do so, i.e.

$$
\sum_{\mathcal{S}} \hat{k}_{i}(z)=\sum_{\mathcal{S}} k_{i},
$$

where all momenta are taken as outgoing. This condition must only hold for the sum over the full set of shifted momenta $\mathcal{S}$, i.e. there should be no subset where (3.4) is satisfied on its own. 
- For all possible "factorization channels", i.e. all decompositions of the set of external momenta $\mathcal{P}$ into two subsets $\mathcal{P}=\mathcal{F} \cup \mathcal{F}^{\prime}$, the sum over momenta in each subset is deformed by a light-like vector,

$$
\hat{K}_{\mathcal{F}}(z)=\sum_{\mathcal{F}} \hat{k}_{i}(z)=K_{\mathcal{F}}+z Q_{\mathcal{F}}, \quad Q_{\mathcal{F}}^{2}=0,
$$

with

$$
Q_{\mathcal{F}}=\sum_{\mathcal{S} \cap \mathcal{F}} \delta k_{i}=-\sum_{\mathcal{S} \cap \mathcal{F}^{\prime}} \delta k_{i}
$$

This condition is not necessary (see e.g. [5]) but chosen here to simplify the discussion. Shifts with $Q_{\mathcal{F}}^{2} \neq 0$ do not lead to advantages for our purposes, as discussed in appendix $\mathrm{C}$.

Since poles of Feynman diagrams arise solely through propagators, only simple poles in $z$ appear for Born amplitudes for the above properties of the shift. The integral of the function $A(z) / z$ over a circle with $|z| \rightarrow \infty$ is given by the sum of the residues at the poles, $z_{\mathcal{F}}$ and the residue at $z=0$, which gives the physical amplitude,

$$
\frac{1}{2 \pi \mathrm{i}} \oint \frac{A(z)}{z}=A(0)+\sum_{\operatorname{poles} z_{\mathcal{F}}} \operatorname{Res}_{z_{\mathcal{F}}} \frac{A(z)}{z} .
$$

Factorization properties of Born amplitudes imply that the $n$-point amplitude at the poles $z_{\mathcal{F}}$ factorizes into lower-multiplicity amplitudes according to ${ }^{1}$

$$
\lim _{z \rightarrow z_{\mathcal{F}}} A(z)=\sum_{s} A_{\mathcal{F}}\left(\ldots \hat{\Phi}_{\mathcal{F}}^{s}\right) \frac{\mathrm{i}}{K_{\mathcal{F}}^{2}+2 z K_{\mathcal{F}} \cdot Q_{\mathcal{F}}-M_{\mathcal{F}}^{2}} A_{\mathcal{F}^{\prime}}\left(\hat{\Phi}_{\mathcal{F}^{\prime}}^{-s}, \ldots\right),
$$

where $\Phi_{\mathcal{F}}^{s}$ denotes a generic particle with spin projection $s$, momentum $\hat{K}_{\mathcal{F}}$ and mass $M_{\mathcal{F}}$. In the following, massive vector bosons will be denoted by $W^{s}$, massive fermions with $s= \pm \frac{1}{2}$ by $\psi^{ \pm}$, and $\phi$ denotes both physical scalars or would-be Goldstone bosons. The poles of the complex variable $z$ are located at

$$
z_{\mathcal{F}}=-\frac{K_{\mathcal{F}}^{2}-M_{\mathcal{F}}^{2}}{2 K_{\mathcal{F}} \cdot Q_{\mathcal{F}}}
$$

Provided the condition

$$
\lim _{z \rightarrow \infty} A(z)=0
$$

holds, the left hand side of (3.7) vanishes and the physical scattering amplitude is expressed in terms of lower-point on-shell amplitudes,

$$
A(0)=\sum_{\mathcal{F}} \sum_{s} A_{\mathcal{F}}\left(\ldots \hat{\Phi}_{\mathcal{F}}^{s}\right) \frac{\mathrm{i}}{K_{\mathcal{F}}^{2}-M_{\mathcal{F}}^{2}} A_{\mathcal{F}^{\prime}}\left(\hat{\Phi}_{\mathcal{F}^{\prime}}^{-s}, \ldots\right)
$$

\footnotetext{
${ }^{1}$ If the internal particle $\Phi_{\mathcal{F}}$ is a fermion, a convention-dependent phase factor arises since one of the momenta $K_{\mathcal{F}}$ and $K_{\mathcal{F}^{\prime}}=-K_{\mathcal{F}}$ corresponds to an incoming particle line [23]. Writing the numerator of a fermion propagator in terms of the completeness relation and using the convention (2.26) one finds for $K_{\mathcal{F}}^{0}+K_{\mathcal{F}}^{3}>0$

$$
K_{\mathcal{F}}+M_{\mathcal{F}}=\sum_{s} u\left(K_{\mathcal{F}},-s\right) \bar{u}\left(K_{\mathcal{F}}, s\right)=\sum_{s}\left(-\mathrm{i} v\left(K_{\mathcal{F}^{\prime}},-s\right)\left(\bar{u}\left(K_{\mathcal{F}}, s\right)\right)\right.
$$
}


On the right-hand side the shifted momenta in the set $\mathcal{S}$ and the momentum $\hat{K}_{\mathcal{F}}$ of the internal line are evaluated at the poles (3.9). A prescription for the reference spinors for internal massive particles is defined in section 3.3.

For internal massive vector bosons, gauge invariance implies that the unphysical degrees of freedom, i.e. the would-be Goldstone bosons and the fourth polarization vector $\epsilon^{\mu}(S)=p^{\mu} / M$ cancel at the pole (3.9) so that only the sum over the three physical polarizations needs to be taken in (3.11).

In this section, we derive little-group covariant expressions for the shift of spinor variables and the wave functions of massive spin one-half fermions and vector bosons. However, in order to satisfy the condition (3.10) we will choose a particular spin axis aligned with the shift $[4,23]$. Therefore it must be possible to recover amplitudes for arbitrary spin axes and spin quantum numbers. There are two ways to achieve this:

- Construct the amplitude for a fixed spin state for arbitrary and independent spin axes $n_{q_{i}}$ for all particles. The spin-dependence of the scattering amplitude enters only through the polarization wave functions, while the remaining truncated amplitude is little-group invariant since it can be expressed in terms of momenta, Lorentz tensors and Dirac matrices. Therefore amplitudes for arbitrary spin states can be obtained using the generators $J_{k_{i}}^{ \pm}(2.16)$, which only act on the wave-function of a single leg $i$.

- Construct the amplitudes for all spin states for a particular fixed choice of the spin axes. Results for arbitrary spin axes can be obtained as linear combinations of these results using the finite little-group transformations of the polarization wave functions (2.27) and (2.39).

In the remainder of this section we construct all possible $h$-line shifts of massive particles satisfying the properties (3.2)-(3.5). The large- $z$ behaviour (3.10) is investigated in section 4 while the ability to construct the amplitudes for all spin states is discussed in section 5 .

\subsection{Shifts of massive momenta}

We first consider the shift of a single massive momentum $k$ such that the on-shell condition (3.3) remains satisfied. In the little-group covariant notation, the shift can be defined by introducing pairs of holomorphic and anti-holomorphic spinors $\eta_{\alpha}^{I}$ and $\eta_{\dot{\alpha}, I}$ and deforming the spinor variables according to

$$
k_{\alpha}^{I} \rightarrow \hat{k}_{\alpha}^{I}(z)=k_{\alpha}^{I}+z \eta_{\alpha}^{I}, \quad \quad k_{\dot{\alpha}, I} \rightarrow \hat{k}_{\dot{\alpha}, I}(z)=k_{\dot{\alpha}, I}+z \eta_{\dot{\alpha}, I} .
$$

The on-shell condition can be satisfied by demanding that the normalization conditions (2.5) are not modified by the shift,

$$
\left\langle\hat{k}^{I}(z) \hat{k}^{J}(z)\right\rangle \stackrel{!}{=} m \varepsilon^{I J}, \quad\left[\hat{k}_{I}(z) \hat{k}_{J}(z)\right] \stackrel{!}{=}-m \varepsilon_{I J}
$$

This implies that the holomorphic spinors $\eta_{\alpha}^{I}$ must satisfy the conditions

$$
\left\langle\eta^{I} \eta^{J}\right\rangle=\frac{1}{2}\left\langle\eta^{I} \eta_{I}\right\rangle \varepsilon^{I J}=0, \quad\left\langle k^{[I} \eta^{J]}\right\rangle=\left\langle k^{I} \eta_{I}\right\rangle \varepsilon^{I J}=0
$$


where it was used that any two-dimensional antisymmetric tensor is proportional to the totally antisymmetric symbol. According to the first condition the shift vector factorizes in terms of a light-like Weyl spinor $\eta_{\alpha}$ and a little-group spinor $n^{I}$,

$$
\eta_{\alpha}^{I}=n^{I} \eta_{\alpha}
$$

The second condition then becomes

$$
\varepsilon_{I J} n^{I}\left\langle k^{J} \eta\right\rangle=0
$$

which determines the little-group spinor up to a constant $c$,

$$
n^{I}=c\left\langle\eta k^{I}\right\rangle .
$$

The shift of the anti-holomorphic variable is treated analogously. The general shift of the massive spinor variables is therefore of the form

$$
\hat{k}_{\alpha}^{I}(z)=k_{\alpha}^{I}+z c \eta_{\alpha}\left\langle\eta k^{I}\right\rangle, \quad \hat{k}_{\dot{\alpha}, I}(z)=k_{\dot{\alpha}, I}+z d \eta_{\dot{\alpha}}\left[k_{I} \eta\right] .
$$

It can be checked that the normalization (2.6) is automatically satisfied by the shifted spinors as well,

$$
\hat{k}_{\alpha}^{I}(z) \hat{k}_{\beta, I}(z)=m \varepsilon_{\alpha \beta}, \quad \hat{k}_{\dot{\alpha}}^{I}(z) \hat{k}_{\dot{\beta}, I}(z)=m \varepsilon_{\dot{\alpha} \dot{\beta}} .
$$

This follows from identities such as $\left\langle\eta k^{I}\right\rangle k_{\beta, I}=m \eta_{\beta}$ that result from (2.6). Note that within the spin-axis formalism the shift (3.18) corresponds to shifting both the momentum spinors $k^{b}$ and the reference spinors $q$.

The requirement (3.2) of a linear shift of the momentum allows only a shift of the holomorphic or anti-holomorphic spinors alone, so there are two possible shifts, ${ }^{2}$

$$
\begin{aligned}
& \hat{k}_{\alpha \dot{\alpha}}^{\mathrm{H}}(z)=k_{\alpha \dot{\alpha}}+z c \eta_{\alpha}\left\langle\eta k^{I}\right\rangle k_{\dot{\alpha}, I}=k_{\alpha \dot{\alpha}}+z c \eta_{\alpha}(\langle\eta| \not k)_{\dot{\alpha}}, \\
& \left.\hat{k}_{\alpha \dot{\alpha}}^{\mathrm{A}}(z)=k_{\alpha \dot{\alpha}}+z d k_{\alpha}^{I}\left[k_{I} \eta\right] \eta_{\dot{\alpha}}=k_{\alpha \dot{\alpha}}+z d(\not k \mid \eta]\right)_{\alpha} \eta_{\dot{\alpha}} .
\end{aligned}
$$

The second form of the shifts follows from the decomposition of the momentum (2.3) and makes it clear that these results reproduce the known expressions for the shift of a massive momentum by a light-like vector $[4,18]$. In this form it is also straightforward to take the massless limit by rescaling $c \rightarrow c /\langle\eta k\rangle$ and $d \rightarrow d /[k \eta]$ so one obtains the familiar result

$$
\hat{k}_{\alpha}^{\mathrm{H}}(z)=k_{\alpha}+z c \eta_{\alpha}, \quad \hat{k}_{\dot{\alpha}}^{\mathrm{A}}(z)=k_{\dot{\alpha}}+z d \eta_{\dot{\alpha}} .
$$

For shifts of multiple external momenta discussed in section 3.3, the constants $c$ and $d$ need to be chosen such that the condition of momentum conservation (3.4) is satisfied.

\footnotetext{
${ }^{2}$ The only other way to avoid a quadratic term in $z$ is to fix the shift spinors in terms of the momentum spinors such that $\left\langle\eta k^{I}\right\rangle\left[k_{I} \eta\right]=0$, e.g. $|\eta\rangle \propto\left|k^{2}\right\rangle$ and $\left.\left.\mid \eta\right] \propto \mid k_{1}\right]$. The resulting momentum shift $\hat{k}_{\alpha \dot{\alpha}}(z)=$ $k_{\alpha \dot{\alpha}}+z \tilde{c} k_{\alpha}^{2} k_{\dot{\alpha}, 1}$ can be viewed as a special case of both the generic holomorphic and anti-holomorphic shifts and need not be considered separately.
} 


\subsection{Shifts of wave-functions}

Expressions for the Dirac spinors (2.19) and polarization vectors (2.30) for a shifted momentum can be easily defined by replacing the spinors $k_{\alpha}^{I}$ and $k_{\dot{\alpha}, I}$ with the corresponding shifted quantities (3.18). For the example of the holomorphic shift one obtains the Dirac wave function

$$
\hat{u}^{I, \mathrm{H}}(k, z)=\left(\begin{array}{c}
\hat{k}_{\alpha}^{I}(z) \\
-k^{\dot{\alpha}, I}
\end{array}\right)=u^{I}(k)+z c\left\langle\eta k^{I}\right\rangle\left(\begin{array}{c}
\eta_{\alpha} \\
0
\end{array}\right) .
$$

The shifts of the conjugate and anti-particle spinors are defined in complete analogy. By construction, these spinors satisfy the appropriate equations of motion (2.18), completeness conditions (2.20) and normalization conditions (2.21) for the shifted momentum since the normalization conventions (2.5) and (2.6) are not affected by the shift. Similarly, the polarization vectors

$$
\hat{\epsilon}_{\alpha \dot{\alpha}}^{(I J), \mathrm{H}}(k, z)=\frac{1}{\sqrt{2} m} \hat{k}_{\alpha}^{(I}(z) k_{\dot{\alpha}}^{J)}=\epsilon_{\alpha \dot{\alpha}}^{(I J)}(k)+\frac{z c}{\sqrt{2} m} \eta_{\alpha}\left\langle\eta k^{(I}\right\rangle k_{\dot{\alpha}}^{J)}
$$

satisfy the transversality condition (2.31), the normalization (2.32) and the completeness relation (2.33) for the shifted momentum. The corresponding results for the anti-holomorphic shift are

$$
\hat{u}^{I, \mathrm{~A}}(k, z)=u^{I}(k)-z d\left[k_{I} \eta\right]\left(\begin{array}{c}
0 \\
\eta^{\dot{\alpha}}
\end{array}\right),
$$

and

$$
\hat{\epsilon}_{\alpha \dot{\alpha}}^{(I J), \mathrm{A}}(k, z)=\frac{1}{\sqrt{2} m} k_{\alpha}^{(I} \hat{k}_{\dot{\alpha}}^{J)}(z)=\epsilon_{\alpha \dot{\alpha}}^{(I J)}(k)+\frac{z d}{\sqrt{2} m} k_{\alpha}^{(I}\left[k^{J)} \eta\right] \eta_{\dot{\alpha}} .
$$

These results provide little-group covariant expressions for shifted Dirac spinors and polarization vectors. However, in general the spinors and polarization vectors for all spin orientations receive a linear shift, which is not desirable in the discussion of the scaling of the amplitude $A(z)$ for $z \rightarrow \infty$. Nevertheless, the little-group covariant form of the shift (3.18) was recently used for a BCFW shift of one massive and one massless leg [30]. In this paper we focus on purely massive shifts and leave a systematic analysis of the large- $z$ behaviour of this type of shift for future work.

\subsubsection{Choice of spin axis}

Using a suitable choice of spin axis aligned with the shift spinors $\eta_{\alpha}, \eta_{\dot{\alpha}}$ it is possible to simplify the shifted wave functions to a form similar to the massless case [4] so that they stay $z$-independent for some spin quantum numbers. ${ }^{3}$ To this end, it is useful to choose the holomorphic reference spinor in the light-cone decomposition (2.8) of a holomorphically shifted momentum $\hat{k}_{i}^{\mathrm{H}}(z)$ as

$$
q_{i, \alpha}=\eta_{i, \alpha},
$$

\footnotetext{
${ }^{3}$ Alternatively, one could consider eliminating holomorphic or anti-holomorphic spinor variables using the on-shell condition (2.2) and the Dirac equation (2.7) as advocated in [11] (see also a related discussion at the Lagrangian level [31]). This corresponds to the introduction of higher-dimensional operators and moves the effect of the shift from the wave functions to the truncated amplitude. This does not appear to simplify the study of the $z \rightarrow \infty$ behaviour but may deserve further study.
} 
while for an anti-holomorphically shifted momenta $\hat{k}_{j}^{\mathrm{A}}(z)$ the choice

$$
q_{j, \dot{\alpha}}=\eta_{j, \dot{\alpha}}
$$

is made. In this way only the light-cone projected momentum spinors are shifted,

$$
\begin{array}{ll}
\hat{k}_{i, \alpha}^{\mathrm{H}}(z)=k_{i, \alpha}^{\mathrm{b}}+z c_{i} \eta_{i, \alpha}, & \hat{k}_{i, \dot{\alpha}}^{\mathrm{H}}(z)=k_{i, \dot{\alpha}}^{\mathrm{b}}, \\
\hat{k}_{i, \alpha}^{\mathrm{A}}(z)=k_{i, \alpha}^{b}, & \hat{k}_{j, \dot{\alpha}}^{\mathrm{b}}(z)=k_{j, \dot{\alpha}}^{b}+z d_{j} \eta_{j, \dot{\alpha}},
\end{array}
$$

where the rescaling $c_{i} \rightarrow c_{i} /\left\langle\eta_{i} k_{i}^{b}\right\rangle$ and $d_{j} \rightarrow d_{j} /\left[k_{j}^{b} \eta_{j}\right]$ was performed. The remaining reference spinors $q_{i, \dot{\alpha}}$ and $q_{j, \alpha}$ are still arbitrary at this stage. With this choice, only the Dirac spinor with negative spin is affected by the holomorphic shift, as in the massless case,

$$
\hat{u}^{\mathrm{H}}\left(k_{i}, \frac{1}{2}\right)=\left(\begin{array}{c}
\frac{m}{\left\langle k_{i}^{b} \eta_{i}\right\rangle} \eta_{i, \alpha} \\
k_{i, \dot{\alpha}}^{b}
\end{array}\right), \quad \hat{u}^{\mathrm{H}}\left(k_{i},-\frac{1}{2}\right)=\left(\begin{array}{c}
k_{i, \alpha}^{\mathrm{b}}+z c_{i} \eta_{i, \alpha} \\
-\frac{m}{\left[q_{i} k_{i}^{b}\right]} q_{i, \dot{\alpha}}
\end{array}\right) .
$$

In the same way, only the polarization vector with negative spin and the longitudinal polarization are shifted,

$$
\begin{aligned}
\hat{\epsilon}_{\alpha \dot{\alpha}}^{\mathrm{H}}\left(k_{i},+\right) & =\sqrt{2} \frac{\eta_{i, \alpha} k_{i, \dot{\alpha}}^{\mathrm{b}}}{\left\langle\eta_{i} k_{i}^{\mathrm{b}}\right\rangle}, \quad \hat{\epsilon}_{\alpha \dot{\alpha}}^{\mathrm{H}}\left(k_{i},-\right)=\sqrt{2} \frac{\left(k_{i, \alpha}^{b}+z c_{i} \eta_{i, \alpha}\right) q_{i, \dot{\alpha}}}{\left[k_{i}^{b} q_{i}\right]}, \\
\hat{\epsilon}_{\alpha \dot{\alpha}}^{\mathrm{H}}\left(k_{i}, 0\right) & =\frac{1}{m}\left(\left(k_{i, \alpha}^{\mathrm{b}}+z c_{i} \eta_{i, \alpha}\right) k_{i, \dot{\alpha}}^{b}-\frac{m^{2}}{\left\langle\eta_{i} k_{i}^{b}\right\rangle\left[k_{i}^{b} q_{i}\right]} \eta_{i, \alpha} q_{i, \dot{\alpha}}\right) .
\end{aligned}
$$

For the anti-holomorphic shift, only the Dirac spinors with positive spin are affected,

$$
\hat{u}^{\mathrm{A}}\left(k_{j}, \frac{1}{2}\right)=\left(\begin{array}{c}
\frac{m}{\left\langle k_{j}^{\mathrm{b}} q_{j}\right\rangle} q_{j, \alpha} \\
k_{j, \dot{\alpha}}^{b}+z d_{j} \eta_{j, \dot{\alpha}}
\end{array}\right), \quad \hat{u}^{\mathrm{A}}\left(k_{j},-\frac{1}{2}\right)=\left(\begin{array}{c}
k_{j, \alpha}^{\mathrm{b}} \\
-\frac{m}{\left[\eta_{j} k_{j}^{\mathrm{b}]}\right]} \eta_{j, \dot{\alpha}}
\end{array}\right),
$$

while for massive vector bosons again also the longitudinal polarization vector is shifted in addition to the positive spin,

$$
\begin{aligned}
\hat{\epsilon}_{\alpha \dot{\alpha}}^{\mathrm{A}}\left(k_{j},+\right) & =\sqrt{2} \frac{q_{j, \alpha}\left(k_{j, \dot{\alpha}}^{\mathrm{b}}+z d_{j} \eta_{j, \dot{\alpha}}\right)}{\left\langle q_{j} k_{j}^{\mathrm{b}}\right\rangle}, \quad \hat{\epsilon}_{\alpha \dot{\alpha}}^{\mathrm{A}}\left(k_{j},-\right)=\sqrt{2} \frac{k_{j, \alpha}^{\mathrm{b}} \eta_{j, \dot{\alpha}}}{\left[k_{j}^{\mathrm{b}} \eta_{j}\right]}, \\
\hat{\epsilon}_{\alpha \dot{\alpha}}^{\mathrm{A}}\left(k_{j}, 0\right) & =\frac{1}{m}\left(k_{j, \alpha}^{\mathrm{b}}\left(k_{j, \dot{\alpha}}^{\mathrm{b}}+z d_{j} \eta_{j, \dot{\alpha}}\right)-\frac{m^{2}}{\left\langle q_{j} k_{j}^{\mathrm{b}}\right\rangle\left[k_{j}^{\mathrm{b}} \eta_{j}\right]} q_{j \alpha} \eta_{j, \dot{\alpha}}\right) .
\end{aligned}
$$

\subsection{Multi-line shifts}

To construct complex deformations of scattering amplitudes, the set $\mathcal{S}$ of shifted momenta is split into two subsets, $\mathcal{S}=\mathcal{H} \cup \mathcal{A}$, where a holomorphic shift (3.20) is performed for a subset of momenta $k_{i} \in \mathcal{H}$ while momenta $k_{j} \in \mathcal{A}$ are deformed by an anti-holomorphic shift (3.21). These candidate shifts must then be constrained so that the conditions (3.4) and (3.5) are satisfied. Momentum conservation (3.4) implies the condition

$$
\begin{aligned}
0=\sum_{\mathcal{H}} \delta k_{i, \alpha \dot{\alpha}}^{\mathrm{H}}+\sum_{\mathcal{A}} \delta k_{j, \alpha \dot{\mathrm{\alpha}}}^{\mathrm{A}} & \left.=\sum_{\mathcal{H}} c_{i} \eta_{i, \alpha}\left(\left\langle\eta_{i}\right| \not k_{i}\right)_{\dot{\alpha}}+\sum_{\mathcal{A}} d_{j}\left(\not k_{j} \mid \eta_{j}\right]\right)_{\alpha} \eta_{j, \dot{\alpha}} \\
& =\sum_{\mathcal{H}} c_{i} \eta_{i, \alpha} k_{i, \dot{\alpha}}^{\mathrm{b}}+\sum_{\mathcal{A}} d_{j} k_{j, \alpha}^{b} \eta_{j, \dot{\alpha}},
\end{aligned}
$$


where the expression in the second line holds for the choice of reference spinors (3.27) and re-scaled coefficients. For generic shift spinors $\eta_{i}$, this identity provides four constraints for the $h$ coefficients $c_{i}$ and $d_{j}$ so that a solution always exists for $h \geq 4$. For the cases $h=2,3$ solutions can be obtained for special choices of shift spinors corresponding to massive generalizations of the BCFW and Risager constructions, as discussed below.

The condition (3.5) implies that the sum of shifted momenta for every factorization channel $\mathcal{F}$ is light-like, i.e. the quantities

$$
\begin{aligned}
Q_{\mathcal{F}, \alpha \dot{\alpha}} & \left.=\sum_{\mathcal{H} \cap \mathcal{F}} c_{i} \eta_{i, \alpha}\left(\left\langle\eta_{i}\right| \not k_{i}\right)_{\dot{\alpha}}+\sum_{\mathcal{A} \cap \mathcal{F}} d_{j}\left(\not k_{j} \mid \eta_{j}\right]\right)_{\alpha} \eta_{j, \dot{\alpha}} \\
& =\sum_{\mathcal{H} \cap \mathcal{F}} c_{i} \eta_{i, \alpha} k_{i, \dot{\alpha}}^{b}+\sum_{\mathcal{A} \cap \mathcal{F}} d_{j} k_{j, \alpha}^{b} \eta_{j, \dot{\alpha}}
\end{aligned}
$$

must factorize into a product of two-component spinors, $Q_{\mathcal{F}, \alpha \dot{\alpha}}=Q_{\mathcal{F}, \alpha} Q_{\mathcal{F}, \dot{\alpha}}$, for all choices of $\mathcal{F}$. Since the number of factorization channels in general exceeds the number $h-4$ of the remaining free coefficients, this requires special choices of the shift spinors $\eta_{i}$. Analogously to the massless case considered in [5] this leaves two possibilities: Generalizations of the construction of Risager [25] where only holomorphic or anti-holomorphic shifts are performed or generalizations of the BCFW construction by performing a holomorphic shift of $h-1$ legs and an anti-holomorphic shift for one leg, or vice versa. The extension of these constructions to the massive case are discussed in sections 3.3.2 and 3.3.3, respectively. The generalization of the original two-line BCFW shift to the massive case needs to be treated separately and is discussed in section 3.3.1. Shifts where $l>1$ lines are shifted holomorphically and $h-l>1$ lines anti-holomorphically lead to factorization channels with $Q_{\mathcal{F}}^{2} \neq 0$ and are discussed in appendix C.

In the application of the recursion relation, also the spin states of the internal particle $\Phi_{\mathcal{F}}^{s}$ in the factorized amplitudes (3.8) need to be defined. It is useful to choose the reference spinors in terms of the factorized spinors of the internal shift (3.35),

$$
q_{\mathcal{F}, \alpha}=Q_{\mathcal{F}, \alpha}, \quad q_{\mathcal{F}, \dot{\alpha}}=Q_{\mathcal{F}, \dot{\alpha}},
$$

which implies the light-cone decomposition of the internal momentum

$$
K_{\mathcal{F}, \alpha \dot{\alpha}}=K_{\mathcal{F}, \alpha}^{b} K_{\mathcal{F}, \dot{\alpha}}^{b}+\frac{K_{\mathcal{F}}^{2}}{2\left(K_{\mathcal{F}} \cdot Q_{\mathcal{F}}\right)} Q_{\mathcal{F}, \alpha} Q_{\mathcal{F}, \dot{\alpha}}
$$

This choice results in a simple expression for the shifted internal momentum,

$$
\hat{K}_{\mathcal{F}, \alpha \dot{\alpha}}(z)=K_{\mathcal{F}, \alpha}^{b} K_{\mathcal{F}, \dot{\alpha}}^{b}+\left(\frac{M_{\mathcal{F}}^{2}}{2\left(K_{\mathcal{F}} \cdot Q_{\mathcal{F}}\right)}+\left(z-z_{\mathcal{F}}\right)\right) Q_{\mathcal{F}, \alpha} Q_{\mathcal{F}, \dot{\alpha}},
$$

which has been expressed in such a way that the on-shell condition,

$$
\hat{K}_{\mathcal{F}}^{2}\left(z_{\mathcal{F}}\right)=M_{\mathcal{F}}^{2}
$$

at the pole position (3.9) is manifestly satisfied. Note the light-cone projected momentum is not affected by the shift, i.e. $\hat{K}_{\mathcal{F}}^{b}=K_{\mathcal{F}}^{b}$. The wave-functions of the internal particles can then be defined in terms of the reference spinors (3.36) and the momentum spinors $K_{\mathcal{F}}^{b}$. 


\subsubsection{Two-line BCFW shifts}

For a two-line BCFW-type shift of massive lines, a solution to the on-shell condition (3.3) and momentum conservation (3.34) was constructed in [23] using the fact that two massive momenta $k_{i / j}$ can be expressed in terms of two light-like vectors $l_{i / j}$ as

$$
k_{i}=l_{i}+\alpha_{j} l_{j}, \quad k_{j}=\alpha_{i} l_{i}+l_{j},
$$

with the coefficients

$$
\alpha_{j}=\frac{2 k_{i} \cdot k_{j}-\operatorname{sgn}\left(2 k_{i} \cdot k_{j}\right) \sqrt{\Delta}}{2 k_{j}^{2}}, \quad \alpha_{i}=\frac{2 k_{i} \cdot k_{j}-\operatorname{sgn}\left(2 k_{i} \cdot k_{j}\right) \sqrt{\Delta}}{2 k_{i}^{2}},
$$

and

$$
\Delta=\left(2 k_{i} \cdot k_{j}\right)^{2}-4 k_{i}^{2} k_{j}^{2} .
$$

A two-line shift with the shift spinors

$$
\eta_{\alpha}=l_{j, \alpha}, \quad \eta_{\dot{\alpha}}=l_{i, \dot{\alpha}},
$$

leads to the momentum shifts

$$
\hat{k}_{i, \alpha \dot{\alpha}}^{\mathrm{H}}(z)=k_{i, \alpha \dot{\alpha}}+z l_{j, \alpha} l_{i, \dot{\alpha}}, \quad \hat{k}_{j, \alpha \dot{\alpha}}^{\mathrm{A}}(z)=k_{j, \alpha \dot{\alpha}}-z l_{j, \alpha} l_{i, \dot{\alpha}},
$$

which manifestly have the desired properties (3.4) and (3.5). This result was recently also obtained from the little-group point of view in [32]. Making the choice (3.43) in the results obtained in section 3.2 provides shifted Dirac spinors (3.23) and polarization vectors (3.24) for arbitrary little-group frames. However, eliminating the $z$-dependence in some of the external wave-functions by fixing the spin axes according to (3.27),

$$
q_{i, \alpha}=\eta_{\alpha}=l_{j, \alpha}, \quad q_{j, \dot{\alpha}}=\eta_{\dot{\alpha}}=l_{i, \dot{\alpha}}
$$

reproduces the definition of shifted Dirac spinors in [23]. Also the prescription (3.36) for the reference spinors of the internal line reproduces the choice of [23],

$$
q_{\mathcal{F}, \alpha}=\eta_{\alpha}=l_{j, \alpha}, \quad q_{\mathcal{F}, \dot{\alpha}}=\eta_{\dot{\alpha}}=l_{i, \dot{\alpha}} .
$$

\subsubsection{Multi-line Risager-type shifts}

In the Risager-type solution to the light-cone condition (3.35) for all factorization channels, all shifts are either exclusively holomorphic or anti-holomorphic and shift spinors $\eta_{i}$ are chosen identical. In the holomorphic case the shift of the spinors is therefore given by

$$
\hat{k}_{i, \alpha}^{I, \mathrm{H}}(z)=k_{i, \alpha}^{I}+z c_{i} \eta_{\alpha}\left\langle\eta k_{i}^{I}\right\rangle, \quad \hat{k}_{i, \dot{\alpha}, I}^{\mathrm{H}}(z)=k_{i, \dot{\alpha}, I},
$$

so that the shift of the momentum of internal propagators factorizes according to

$$
Q_{\mathcal{F}, \alpha \dot{\alpha}}=\eta_{\alpha} \sum_{\mathcal{S} \cap \mathcal{F}} c_{i}\left(\langle\eta| \not k_{i}\right)_{\dot{\alpha}} \equiv \eta_{\alpha} Q_{\mathcal{F}, \dot{\alpha}}
$$


Choosing the spin axis according to (3.27) implies that the same reference spinor $q_{i, \alpha}=\eta_{\alpha}$ is used for all shifted particles. This simplifies the shifted momentum spinors to

$$
\hat{k}_{i, \alpha}^{\mathrm{bH}}(z)=k_{i, \alpha}^{b}+z c_{i} \eta_{\alpha}, \quad \hat{k}_{i, \dot{\alpha}}^{b \mathrm{H}}(z)=k_{i, \dot{\alpha}}^{b},
$$

and shifted Dirac spinors and polarization vectors are given by (3.30) and (3.31). The light-cone projection of the internal momentum is defined using the reference spinors

$$
q_{\mathcal{F}, \alpha}=\eta_{\alpha}, \quad q_{\mathcal{F}, \dot{\alpha}}=Q_{\mathcal{F}, \dot{\alpha}}=\sum_{\mathcal{S} \cap \mathcal{F}} c_{i} k_{i, \dot{\alpha}}^{b}
$$

The condition of momentum conservation (3.34) becomes

$$
0=\sum_{\mathcal{S}} c_{i} k_{i, \dot{\alpha}}^{b}
$$

For three shifted momenta, $i \in\left\{i_{1}, i_{2}, i_{3}\right\}$ the Schouten identity implies the solution

$$
c_{i_{1}}=\left[k_{i_{2}}^{b} k_{i_{3}}^{b}\right], \quad c_{i_{2}}=\left[k_{i_{3}}^{b} k_{i_{1}}^{b}\right], \quad c_{i_{3}}=\left[k_{i_{1}}^{b} k_{i_{2}}^{b}\right],
$$

in complete analogy to the massless case [25]. In general, a system of $h$ equations for the coefficients can be obtained by contracting with all of the $k_{i, \dot{\alpha}}^{b}$ spinors of the shifted legs. However, the system is under-determined due to the Schouten identity. In our construction of scattering amplitudes we will require four-line and five-line shifts, for which solutions can be written as

$$
c_{i_{1}}=\left[k_{i_{2}}^{b} k_{i_{3}}^{b}\right], \quad c_{i_{2}}=\left[k_{i_{3}}^{b} k_{i_{1}}^{b}\right]+\left[k_{i_{3}}^{b} k_{i_{4}}^{b}\right], \quad c_{i_{3}}=\left[k_{i_{1}}^{b} k_{i_{2}}^{b}\right]+\left[k_{i_{4}}^{b} k_{i_{2}}^{b}\right], \quad c_{i_{4}}=\left[k_{i_{2}}^{b} k_{i_{3}}^{b}\right],
$$

and

$$
c_{i_{1}}=\left[k_{i_{2}}^{b} k_{i_{3}}^{b}\right], \quad c_{i_{2}}=\left[k_{i_{3}}^{b} k_{i_{1}}^{b}\right], \quad c_{i_{3}}=\left[k_{i_{1}}^{b} k_{i_{2}}^{b}\right]+\left[k_{i_{4}}^{b} k_{i_{5}}^{b}\right], \quad c_{i_{4}}=\left[k_{i_{5}}^{b} k_{i_{3}}^{b}\right], \quad c_{i_{5}}=\left[k_{i_{3}}^{b} k_{i_{4}}^{b}\right] .
$$

The anti-holomorphic Risager-type shift is given analogously by (3.29) with the choice of reference spinor $q_{j, \dot{\alpha}}=\eta_{\dot{\alpha}}$ for all shifted lines, and with corresponding solutions for the coefficients $d_{j}$.

\subsubsection{Multi-line BCFW-type shifts}

Generalizations of the BCFW construction are obtained by performing an anti-holomorphic shift for one leg $k_{j} \in \mathcal{A}$ and holomorphic shifts of $h-1$ legs $k_{i} \in \mathcal{H}$, with all shift spinors chosen identical,

$$
\begin{array}{llrl}
\hat{k}_{i, \alpha}^{I, \mathrm{H}}(z) & =k_{i, \alpha}^{I}+z c_{i} \eta_{\alpha}\left\langle\eta k_{i}^{I}\right\rangle, & & \hat{k}_{i, \dot{\alpha}, I}^{\mathrm{H}}(z)=k_{i, \dot{\alpha}, I}, \\
\hat{k}_{j, \alpha}^{I, \mathrm{~A}}(z)=k_{j, \alpha}^{I}, & \hat{k}_{j, \dot{\alpha}, I}^{\mathrm{A}}(z)=k_{j, \dot{\alpha}, I}+z d_{j} \eta_{\dot{\alpha}}\left[k_{j, I} \eta\right] .
\end{array}
$$

The condition of light-like shifts $Q_{\mathcal{F}}$ of the internal momenta (3.35) requires the choice

$$
\left.\eta_{\alpha} \propto\left(\not k_{j} \mid \eta\right]\right)_{\alpha},
$$


so that the shift factorizes for all factorization channels,

$$
\left.Q_{\mathcal{F}, \alpha \dot{\alpha}}=\left(\not \aleph_{j} \mid \eta\right]\right)_{\alpha} Q_{\mathcal{F}, \dot{\alpha}}
$$

The choice of spin axis (3.27) implies that all legs in $\mathcal{H}$ share the same holomorphic reference spinor. After re-scaling the coefficients $c_{i}$ and $d_{j}$, the shift and reference spinors become

$$
\eta_{\alpha}=q_{i, \alpha}=k_{j, \alpha}^{b}, \quad \eta_{\dot{\alpha}}=q_{j, \dot{\alpha}} .
$$

The anti-holomorphic reference spinors $q_{i, \dot{\alpha}}$ of the legs in $\mathcal{H}$ and all reference spinors of particle $j$ are kept arbitrary. The generalized BCFW shift therefore takes the simple form

$$
\begin{array}{ll}
\hat{k}_{i, \alpha}^{\mathrm{bH}}(z)=k_{i, \alpha}^{\mathrm{b}}+z c_{i} k_{j, \alpha}^{\mathrm{b}}, & \hat{k}_{i, \dot{\alpha}}^{\mathrm{bH}}(z)=k_{i, \dot{\alpha}}^{\mathrm{b}}, \\
\hat{k}_{j, \alpha}^{\mathrm{b}}(z)=k_{j, \alpha}^{b}, & \hat{k}_{j, \dot{\alpha}}^{\mathrm{A}}(z)=k_{j, \dot{\alpha}}^{b}+z d_{j} q_{j, \dot{\alpha}} .
\end{array}
$$

The shifted Dirac spinors and polarization vectors are given by (3.30) and (3.31) with $\eta_{\alpha}=k_{j, \alpha}^{b}$ for the holomorphic lines and by (3.32) and (3.33) with $\eta_{\dot{\alpha}}=q_{j, \dot{\alpha}}$ for the antiholomorphic line. According to (3.36) the light-cone projection of the internal momentum is defined using the reference spinors

$$
q_{\mathcal{F}, \alpha}=k_{j, \alpha}^{b}, \quad q_{\mathcal{F}, \dot{\alpha}}=Q_{\mathcal{F}, \dot{\alpha}}=\sum_{\mathcal{H} \cap \mathcal{F}} c_{i} k_{i, \dot{\alpha}}^{b}+\sum_{\mathcal{A} \cap \mathcal{F}} d_{j} q_{j, \dot{\alpha}} .
$$

The condition of momentum conservation (3.34) reads

$$
0=\sum_{\mathcal{H}} c_{i} k_{i, \dot{\alpha}}^{b}+d_{j} q_{j, \dot{\alpha}}
$$

In the case of three shifted momenta, $i \in\left\{i_{1}, i_{2}\right\}$, a solution is found with help of the Schouten identity

$$
c_{i_{1}}=\left[k_{i_{2}}^{b} q_{j}\right], \quad c_{i_{2}}=\left[q_{j} k_{i_{1}}^{b}\right], \quad d_{j}=\left[k_{i_{1}}^{b} k_{i_{2}}^{b}\right] .
$$

The solutions for four-line (five-line) shifts can be obtained from the expressions (3.53) and (3.54) for the Risager shift by the replacement $c_{i_{4}} \rightarrow d_{j}$ and $k_{i_{4}} \rightarrow q_{j}\left(c_{i_{5}} \rightarrow d_{j}\right.$ and $\left.k_{i_{5}} \rightarrow q_{j}\right)$.

The BCFW-type shift with one holomorphic line $k_{i}$ and $h-1$ anti-holomorphic lines $k_{j}$ can similarly be brought to the form

$$
\begin{array}{ll}
\hat{k}_{i, \alpha}^{\mathrm{bH}}(z)=k_{i, \alpha}^{\mathrm{b}}+z c_{i} q_{i, \alpha}, & \hat{k}_{i, \dot{\alpha}}^{\mathrm{bH}}(z)=k_{i, \dot{\alpha}}^{b}, \\
\hat{k}_{j, \alpha}^{b \mathrm{~A}}(z)=k_{j, \alpha}^{b}, & \hat{k}_{j, \dot{\alpha}}^{\mathrm{bA}}(z)=k_{j, \dot{\alpha}}^{b}+z d_{j} k_{i, \dot{\alpha}}^{b},
\end{array}
$$

with the shift and reference spinors

$$
\eta_{\alpha}=q_{i, \alpha}, \quad \eta_{\dot{\alpha}}=q_{j, \dot{\alpha}}=k_{i, \dot{\alpha}}^{b} .
$$

The light-cone decomposition of internal momenta is performed using the reference spinors

$$
q_{\mathcal{F}, \alpha}=Q_{\mathcal{F}, \alpha}=\sum_{\mathcal{H} \cap \mathcal{F}} c_{i} q_{i, \alpha}+\sum_{\mathcal{A} \cap \mathcal{F}} d_{j} k_{j, \alpha}, \quad q_{\mathcal{F}, \dot{\alpha}}=k_{i, \dot{\alpha}}^{b}
$$




\section{Large- $z$ behaviour of amplitudes}

In this section we obtain bounds on the large- $z$ behaviour of the complex continuation of $n$-point scattering amplitudes,

$$
\lim _{z \rightarrow \infty} A_{n}(z) \sim z^{\gamma}
$$

under the shifts constructed in section 3. Since $z$ enters only through momenta and external wave-functions, which are all deformed linearly, the exponent $\gamma$ must be an integer, so the criterion $\gamma<0$ ensures the validity of the condition (3.10). The complex continuation of an $n$-particle scattering amplitude with $h$ shifted external particles can be written in terms of "skeleton amplitudes" $\tilde{A}_{h, b}(z)$ describing the scattering of the shifted particles, dressed by insertions of "background" subdiagrams $B_{i}$ with the unshifted external legs,

$$
A_{n}(z)=\sum_{b=1}^{n-h} \sum_{\text {diagrams }} \tilde{A}_{h, b}(z) \prod_{i=1}^{b} B_{i},
$$

see figure 1 for illustration. More precisely, the background subamplitudes are defined in terms of off-shell currents $\mathcal{B}_{i}$, which are given by the off-shell amplitude with $b_{i}$ external on-shell legs and one off-shell leg with attached propagator. The numerator of the propagator of the off-shell leg can be written using a completeness relation in terms of suitably off-shell continued polarization spinors or vectors and possible additional off-shell terms (see e.g. [33]), so that the background currents take the schematic form

$$
\mathcal{B}_{i}=\sum_{s} \chi_{i}(s) \frac{1}{P_{i}^{2}-M^{2}} \chi_{i}^{\dagger}(-s) \tilde{B}_{i} \equiv \sum_{s} \chi_{i}(s) B_{i}(-s),
$$

where the sum over $s$ extends beyond the physical polarizations in the off-shell case. In (4.2) the spin sums are implicit and the polarization factors $\chi_{i}$ are included in the definition of the skeleton amplitudes $\tilde{A}_{h, b}$, so that these contain no open spinor or vector indices. The mass dimension of the background subamplitudes is given by

$$
\left[B_{i}\right]=4-\left(b_{i}+1\right)-2=1-b_{i},
$$

where the term -2 arises from the propagator denominator of the off-shell leg. This split into skeleton and background amplitudes is similar to the background field analysis in [5], although the precise definitions of the background insertions are somewhat different and lead to different mass dimensions of the skeleton and background amplitudes compared to our diagrammatic definition.

The skeleton amplitudes with $h$ shifted lines and $b$ background insertions can be broken down into building blocks according to

$$
\tilde{A}_{h, b}(z)=\frac{N_{h, b}(z)}{D_{h, b}(z)} \prod_{a} g_{a} \prod_{\mathcal{S}_{\psi}} \hat{u}(z) \prod_{\mathcal{S}_{W}} \hat{\epsilon}(z) \prod_{i=1}^{b} \chi_{i},
$$

where $g_{a}$ are coupling constants, $D_{h, b}$ is the product of propagator denominators and $N_{h, b}$ the corresponding numerator function arising from vertex factors and propagator numerators. The set of shifted fermion and vector boson lines is denoted by $\mathcal{S}_{\psi}$ and $\mathcal{S}_{W}$, respectively. The skeleton amplitude is connected, i.e. all propagators in $\tilde{A}_{h, b}$ are $z$-dependent, 


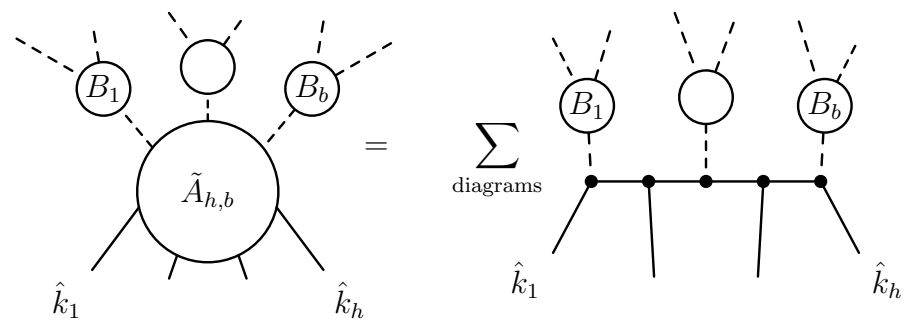

Figure 1. Illustration of the skeleton and background amplitudes appearing in (4.2). The shifted momentum flows through solid lines while dashed lines denote background lines.

since the shift is assumed to satisfy the property that the condition of momentum conservation (3.4) does not hold for a subset of the shifted legs.

Dimensional analysis relates the dimension of the scattering amplitude in four spacetime dimensions to the mass dimensions of the objects in the ansatz (4.5)

$$
\left[\tilde{A}_{h, b}\right]=4-(h+b)=[g]+\left[N_{h, b}\right]-\left[D_{h, b}\right]+\frac{1}{2} h_{\psi}+\frac{1}{2} b_{\psi} .
$$

The term $[g]$ denotes the dimension of the product of coupling constants, $h_{\Phi}$ denotes the number of shifted external legs of particle type $\Phi$ and $b_{\Phi}$ the corresponding background attachments. Similarly, the number of holomorphically or anti-holomorphically shifted particles will be denoted by $h_{\Phi}^{\mathrm{H}}$ and $h_{\Phi}^{\mathrm{A}}$.

The scaling exponent of the skeleton amplitudes, $\lim _{z \rightarrow \infty} \tilde{A}_{h, b}(z) \sim z^{\gamma_{h, b}}$, can be decomposed as

$$
\gamma_{h, b}=\gamma_{N}-\gamma_{D}
$$

where $\gamma_{D}$ arises from the propagator denominators and $\gamma_{N}$ arises from the flow of $z$ through momentum-dependent numerators of Feynman diagrams and from external wave functions. Since the background subamplitudes are $z$-independent, the behaviour of the full amplitude for $z \rightarrow \infty$ is determined by the worst scaling among the skeleton amplitudes,

$$
\gamma=\max \gamma_{h, b}
$$

The criterion $\gamma_{h, b}<0$ is therefore sufficient to establish the condition (3.10).

\subsection{Scaling of external states and Goldstone-boson equivalence}

With the choice of spin axis described in section 3.2.1, the large-z behaviour of the fermion spinors for holomorphic and anti-holomorphic shifts is given by

$$
\begin{aligned}
& \hat{u}^{\mathrm{H}}(k, s) \sim z^{-s+\frac{1}{2}}, \\
& \hat{u}^{\mathrm{A}}(k, s) \sim z^{s+\frac{1}{2}},
\end{aligned}
$$

while the vector-boson polarizations behave as

$$
\begin{aligned}
& \hat{\epsilon}^{\mathrm{H}}(k,-) \sim z^{1}, \quad \hat{\epsilon}^{\mathrm{H}}(k, 0) \sim z^{1}, \quad \hat{\epsilon}^{\mathrm{H}}(k,+) \sim z^{0}, \\
& \hat{\epsilon}^{\mathrm{A}}(k,-) \sim z^{0}, \quad \hat{\epsilon}^{\mathrm{A}}(k, 0) \sim z^{1}, \quad \hat{\epsilon}^{\mathrm{A}}(k,+) \sim z^{1} .
\end{aligned}
$$


The naive scaling of the polarization vectors for the "good shifts", $\hat{\epsilon}^{\mathrm{A}}(-)$ and $\hat{\epsilon}^{\mathrm{H}}(+)$, is worse than in the massless case, where a gauge-dependent $1 / z$ pole of polarization vectors improves the scaling. However, using Ward identities it is possible to establish the $1 / z$ suppression of amplitudes for these polarizations also for a gauge choice with $z$-independent polarization vectors [24]. Similarly, gauge cancellations improve the behaviour of amplitudes with longitudinal polarization vectors compared to the naive estimate [4].

For the case of spontaneously broken gauge invariance, the relevant Ward identity relates vector-boson to Goldstone-boson amplitudes [34, 35],

$$
k_{\mu} A^{\mu}(W(k), \ldots)=m_{W} A(\phi(k), \ldots) .
$$

Using the observation that the shift of the longitudinal polarization vector is proportional to the momentum shift,

$$
\hat{\epsilon}_{\alpha \dot{\alpha}}^{\mathrm{H}}(k, 0)-\epsilon_{\alpha \dot{\alpha}}(k, 0)=\frac{1}{m} c z \eta_{\alpha} k_{\dot{\alpha}}^{b}=\frac{1}{m}\left(\hat{k}_{\alpha \dot{\alpha}}^{\mathrm{H}}(z)-k_{\alpha \dot{\alpha}}\right),
$$

and similarly for the anti-holomorphic shift, the identity (4.11) can be used to obtain the relation for amplitudes with shifted longitudinal vector bosons,

$$
\hat{\epsilon}_{\mu}(k, 0) A^{\mu}(W(\hat{k}(z)), \ldots)=A(\phi(\hat{k}(z)), \ldots)+r_{k, \mu} A^{\mu}(W(\hat{k}(z)), \ldots),
$$

with

$$
r_{k, \alpha \dot{\alpha}}=\left(\epsilon_{\alpha \dot{\alpha}}(k, 0)-\frac{k_{\alpha \dot{\alpha}}}{m}\right)=-\frac{m^{2}}{q \cdot k} q_{\alpha} q_{\dot{\alpha}}
$$

The amplitudes on the right-hand side depend on $z$, while the vector $r_{k}$ does not. In the usual application of the Ward identity (4.11) in the context of the Goldstone boson equivalence theorem, the high-energy limit is taken where $r_{k} \sim m / E$ so that the term involving the amputated amplitude $A^{\mu}(W, \ldots)$ is subdominant compared to the Goldstone boson amplitude. Here we consider the $z \rightarrow \infty$ limit where the exact identity (4.13) cannot be simplified further. This is, however, sufficient to see that the scaling of the longitudinal polarization vectors (4.10) overestimates the $z \rightarrow \infty$ behaviour. Note that the Goldstone-boson and the vector-boson amplitude on the right-hand side of (4.13) may have a different behaviour for $z \rightarrow \infty$ and the contraction of the remainder $r_{k}$ with the vector-boson amplitude must be taken into account in the estimate of the large- $z$ behaviour.

The Ward identity (4.11) can also be used to bound the large- $z$ behaviour of amplitudes with vector bosons with positive spin projection in the holomorphic shift $[24,36]$ by noting that the holomorphic shift of the momentum can be written in terms of the positive-helicity polarization vector,

$$
\hat{k}_{\alpha \dot{\alpha}}^{\mathrm{H}}(z)-k_{\alpha \dot{\alpha}}=z c \frac{\left[k^{\mathrm{b}} q\right]}{\sqrt{2}} \hat{\epsilon}_{\alpha \dot{\alpha}}^{\mathrm{H}}(k,+) .
$$

The application of the Ward identity implies

$$
\hat{\epsilon}_{\mu}^{\mathrm{H}}(k,+) A^{\mu}(W(\hat{k}(z)), \ldots)=\frac{1}{z} \frac{\sqrt{2}}{c\left[k^{b} q\right]}\left(m A(\phi(\hat{k}(z)), \ldots)-k_{\mu} A^{\mu}(W(\hat{k}(z)), \ldots)\right) .
$$

An analogous identity holds for $\hat{\epsilon}^{\mathrm{A}}(k,-)$ in the anti-holomorphic shift. 
Altogether these results show that gauge cancellations, as encoded in the Ward identity (4.11), improve the scaling behaviour of massive vector bosons compared to the naive estimates (4.10) so that the effective scaling is determined by the spin projection $s=0, \pm 1$,

$$
\begin{aligned}
& \hat{\epsilon}^{\mathrm{H}}(k, s) \sim z^{-s}, \\
& \hat{\epsilon}^{\mathrm{A}}(k, s) \sim z^{s} .
\end{aligned}
$$

Therefore the effective behaviour of amplitudes for the "good shifts" of vector bosons is identical to the massless case while the longitudinal vector bosons behave like scalars, as intuitively anticipated from Goldstone boson equivalence. The use of the Ward identity is a key place of our analysis where the consequences of spontaneously broken gauge invariance are employed.

\subsection{Propagator scaling}

For internal lines with light-like shifts, every propagator denominator in the skeleton amplitude is linear in $z$ so that

$$
\gamma_{D}=\frac{1}{2}\left[D_{h, b}\right]=d,
$$

where $d$ is the number of propagators in the skeleton amplitude. This can be estimated using the topological identities of tree diagrams

$$
\begin{gathered}
\sum_{n} v_{n}=d+1, \\
\sum_{n} n v_{n}=2 d+e,
\end{gathered}
$$

where $v_{n}$ is the number of vertices with valency $n$ and $e=h+b$ is the number of external legs of the skeleton amplitude. The number of propagators is bounded from above and below in terms of the smallest and highest valencies $n_{\min }$ and $n_{\max }$,

$$
\frac{h+b-n_{\max }}{n_{\max }-2} \leq \gamma_{D} \leq \frac{h+b-n_{\min }}{n_{\min }-2} .
$$

In the following we limit ourselves to a renormalizable SBGT where $n_{\min }=3$ and $n_{\max }=4$ so that

$$
\frac{h+b-4}{2} \leq \gamma_{D} \leq h+b-3 .
$$

This condition can easily be relaxed for the interesting application of on-shell methods in effective-field-theories of SBGTs with higher-dimensional operators [17, 30, 37-39]. In the derivation of the large- $z$ behaviour in this section, only the upper bound in (4.22) enters, so these results are also valid in the presence of higher-dimensional operators.

\subsection{Bounds for generic shifts with $Q_{\mathcal{F}}^{2}=0$}

In addition to the bound on the denominator (4.22), the estimate (4.7) of the large $z$ scaling of the skeleton amplitude requires a bound on $\gamma_{N}$, i.e. on the $z$-dependence of the numerator function $N_{h, b}$ in the ansatz (4.5), contracted with the polarization functions of 
shifted legs and background insertions. Independent of the structure of the shift, the most conservative estimate is obtained from the mass dimension of the numerator function [5], which can be expressed in terms of (4.6). Adding the scaling of the external fermions (4.9) and the improved estimate for vector bosons (4.17) gives the bound

$$
\begin{aligned}
\gamma_{N} & \leq\left[N_{h, b}\right]+\sum_{\mathcal{H}_{\psi}}\left(-s_{i}+\frac{1}{2}\right)+\sum_{\mathcal{A}_{\psi}}\left(s_{j}+\frac{1}{2}\right)-\sum_{\mathcal{H}_{W}} s_{i}+\sum_{\mathcal{A}_{W}} s_{j} \\
& =4-(h+b)-[g]-\frac{b_{\psi}}{2}+\left[D_{h, b}\right]-\sum_{\mathcal{H}} s_{i}+\sum_{\mathcal{A}} s_{j} .
\end{aligned}
$$

For the concrete examples of the extended Risager and BCFW shifts, better estimates can be obtained, as discussed below. Using the estimate of the scaling of the propagator (4.22), a conservative bound for the scaling of the amplitude is obtained,

$$
\begin{aligned}
\gamma & \leq 4-(h+b)-\min [g]-\frac{b_{\psi}}{2}+\gamma_{D}-\sum_{\mathcal{H}} s_{i}+\sum_{\mathcal{A}} s_{j} \\
& \leq 1-\min [g]-\sum_{\mathcal{H}} s_{i}+\sum_{\mathcal{A}} s_{j},
\end{aligned}
$$

where $b_{\psi} \geq 0$ was used. This result agrees with the case $n_{\min }=3$ in the result for the massless case in eq. (26) in [5]. However, the first line of (4.24) shows that this bound can be improved in amplitudes with fermionic background insertions. Note that the Feynman diagrams contributing to the amplitude may have different mass dimension $[g]$ of the product of coupling constants, so the smallest among these values must be taken for the bound in (4.24).

\subsection{Bounds for multi-line Risager and BCFW shifts}

The generic bound (4.24) can be improved by an analysis of the structure of the contraction of the numerator function in the skeleton amplitude (4.5) with the external and background wavefunctions. Compared to the corresponding discussion of the massless case [5], complications for massive particles arise due to the presence of reference spinors and the need to apply Ward identities to bound the scaling of amplitudes with vector bosons. The following analysis does not cover two-line BCFW shifts, where the estimate (4.24) can be used.

Using the definition of the polarization wave functions, the numerator can be written as a polynomial in holomorphic and anti-holomorphic spinor products of momentum spinors and reference spinors. The $z$-dependence in the terms contributing to the skeleton amplitude is of the schematic form

$$
\tilde{A}_{h, b}(z) \sim R_{h, b} \frac{\left\langle\hat{k}_{i}^{b \mathrm{H}}(z) \cdot\right\rangle^{\alpha}\left[\hat{k}_{j}^{b \mathrm{~A}}(z) \cdot\right]^{\beta}}{D_{h, b}(z)} .
$$

The different contributions to the shifted holomorphic and anti-holomorphic spinor products $\left\langle\hat{k}_{i}^{\mathrm{H}}(z) \cdot\right\rangle$ and $\left[\hat{k}_{j}^{\mathrm{A}}(z) \cdot\right]$ will be analyzed for the extended Risager and BCFW-type shifts below. The function $R_{h, b}$ includes unshifted spinor products, particle masses and coupling constants. The analysis will be performed in the 't Hooft-Feynman gauge where 
the numerators of vector-boson propagators are momentum independent. Note that the exponents $\alpha$ and $\beta$ are positive since spinor products in the denominator can only arise from the definitions of the polarization wave functions, which are not shifted and therefore included in the remainder function $R_{h, b}$.

For all the considered shifts the spinor products involving shifted spinors in the skeleton amplitude (4.25) are linear in $z$ so that the large- $z$ scaling of the numerator function is given by $\gamma_{N}=\alpha+\beta$. However, as discussed in section 4.1, Ward identities improve the large- $z$ behaviour for the "good shifts" of vector bosons and for longitudinal bosons in SBGTs compared to naive scaling estimates. Therefore, the estimate of $\alpha$ and $\beta$ should be based on the right-hand sides of (4.13) and (4.16). The explicit factor of $1 / z$ in (4.16) can be taken into account by defining the effective scaling exponent,

$$
\gamma_{N}=\alpha+\beta-h_{W(+)}^{\mathrm{H}}-h_{W(-)}^{\mathrm{A}},
$$

with the numbers $h_{W(+)}^{\mathrm{H}}\left(h_{W(-)}^{\mathrm{A}}\right)$ of holomorphically (anti-holomorphically) shifted vector bosons with positive (negative) spin projection.

\subsubsection{Multi-line Risager-type shifts}

In an extended holomorphic Risager-type shift as constructed in section 3.3.2, the momenta of all particles in the set $\mathcal{S}$ are shifted as (3.49) and the spin axis of all shifted particles is taken as $q_{i, \alpha}=\eta_{\alpha}$. Therefore the only spinor products that are affected by the shift are

$$
\left\langle\hat{k}_{i}^{b \mathrm{H}}(z) \hat{k}_{k}^{b \mathrm{H}}(z)\right\rangle=\left\langle k_{i}^{b} k_{k}^{b}\right\rangle+z\left(c_{i}\left\langle\eta k_{k}^{b}\right\rangle+c_{k}\left\langle k_{i}^{b} \eta\right\rangle\right), \quad\left\langle\hat{k}_{i}^{b \mathrm{H}}(z) \chi\right\rangle=\left\langle k_{i}^{b} \chi\right\rangle+z c_{i}\langle\eta \chi\rangle,
$$

where $\chi$ denotes a generic unshifted spinor, e.g. from background insertions. Due to the choice of spin axes of the shifted particles, products involving the reference spinors, $\left\langle\hat{k}_{i}^{b \mathrm{H}}(z) q_{k}\right\rangle=\left\langle k_{i}^{b} \eta\right\rangle$ are not shifted and accordingly contribute to the remainder function $R_{h, b}$ in (4.25). The same holds for all anti-holomorphic spinor products, so $\beta=0$. Therefore the relevant contributions to the holomorphic spinor products are of the form

$$
\left\langle\hat{k}_{i}^{\mathrm{bH}}(z) \cdot\right\rangle^{\alpha}=\left\langle\hat{k}_{i}^{b \mathrm{H}}(z) \hat{k}_{k}^{b^{\mathrm{H}}}(z)\right\rangle^{\alpha_{1}}\left\langle\hat{k}_{i}^{\mathrm{bH}}(z) \chi\right\rangle^{\alpha_{2}} \text {. }
$$

The most conservative bound is obtained by assuming that the function $R_{h, b}$ does not contain any holomorphic spinors. Since spinor products of shifted holomorphic spinors are linear in $z$, the upper bound on $\gamma_{N}$ is obtained from half of the number of those holomorphic spinors that contribute to $\alpha_{1}$ and $\alpha_{2}$. This receives the following contributions:

- The number of holomorphic spinors in the numerator function $N_{h, b}$, which can be bound by the mass dimension $\left[N_{h, b}\right]$ since every four-momentum in the numerator gives rise to one holomorphic spinor.

- The number of shifted external holomorphic spinors $\hat{k}_{i, \alpha}^{\mathrm{bH}}(z)$, which arise only from the wave functions $\hat{u}_{i}^{\mathrm{H}}\left(-\frac{1}{2}\right)$ and $\hat{\epsilon}_{i}^{\mathrm{H}}(-)$ after applying the Ward identity. 
- The number of shifted vector bosons with positive spin projection. This contribution arises in addition to the explicit term in (4.26) since the term $k_{k, \mu} A^{\mu}(W, \ldots)$ in the Ward-identity (4.16) can give rise to a spinor product $\left\langle\hat{k}_{i}^{\mathrm{bH}}(z) k_{k}^{b}\right\rangle$ in the numerator. ${ }^{4}$

- The number $b_{\psi}+b_{W}$ of effective polarization functions $\chi$ of the background legs, which contain at most one holomorphic spinor in the numerator.

Including all of these contributions gives rise to the estimate

$$
\begin{aligned}
\gamma_{N} & \leq \frac{1}{2}\left(\left[N_{h, b}\right]+b_{\psi}+b_{W}+h_{\psi(-)}+h_{W(-)}-h_{W(+)}\right) \\
& =\frac{1}{2}\left(4-h-\min [g]-b_{\phi}-\frac{b_{\psi}}{2}-\sum_{\mathcal{S}} s_{i}\right)+\gamma_{D},
\end{aligned}
$$

where (4.6) was used with $b=b_{\phi}+b_{\psi}+b_{W}$. We have also simplified $h_{\psi(-)}-\frac{1}{2} h_{\psi}=$ $\frac{1}{2}\left(h_{\psi(-)}-h_{\psi(+)}\right)=-\sum_{\psi} s_{i}$. Therefore the full large- $z$ behaviour of the amplitude can be bound by

$$
\gamma_{\text {Risager }}^{\mathrm{H}}=\gamma_{N}-\gamma_{D} \leq \frac{1}{2}\left(4-h-\min [g]-\sum_{\mathcal{S}} s_{i}\right),
$$

since $b_{\psi}, b_{\phi} \geq 0$. Note that for specific examples the estimate can be improved by taking the concrete structure of the background into account. For the anti-holomorphic Risager shift, this bound holds with the replacement $s_{i} \rightarrow-s_{j}$. These results agree with those for the massless case [5] and for massive all-line shifts [4].

\subsubsection{Multi-line BCFW-type shifts}

In the generalized BCFW shift (3.59), $h-1$ momenta $\hat{k}_{i}^{\mathrm{H}}$ are shifted holomorphically and a single momentum $\hat{k}_{j}^{\mathrm{A}}$ anti-holomorphically with the shift spinors $\eta_{\alpha}=k_{j, \alpha}^{b}$ and arbitrary $\eta_{\dot{\alpha}}=q_{j, \dot{\alpha}}$. The choice of the reference spinors for the shifted legs is given in (3.58). To estimate the number of spinor products with anti-holomorphically shifted spinors in (4.25), note that momentum conservation can be used in the numerator function $N_{h, b}$ to eliminate $\hat{k}_{j}^{\mathrm{A}}$ in favour of the remaining external momenta of the skeleton amplitude. Therefore only the external wave-functions need to be considered. After application of the Ward identity, only $\hat{\epsilon}_{j}^{\mathrm{A}}(+)$ and $\hat{u}_{j}^{\mathrm{A}}\left(+\frac{1}{2}\right)$ contribute,

$$
\beta \leq h_{\psi(+)}^{\mathrm{A}}+h_{W(+)}^{\mathrm{A}} .
$$

The nontrivial holomorphic spinor products of the shifted spinors among themselves and with other spinors are ${ }^{5}$

$$
\begin{aligned}
\left\langle\hat{k}_{i}^{b \mathrm{H}}(z) \hat{k}_{k}^{b \mathrm{H}}(z)\right\rangle & =\left\langle k_{i}^{b} k_{k}^{b}\right\rangle+z\left(c_{i}\left\langle k_{j}^{b} k_{k}^{b}\right\rangle+c_{k}\left\langle k_{i}^{b} k_{j}^{b}\right\rangle\right), \\
\left\langle\hat{k}_{i}^{b \mathrm{H}}(z) \chi\right\rangle & =\left\langle k_{i}^{\mathrm{b}} \chi\right\rangle+z c_{i}\left\langle k_{j}^{\mathrm{b}} \chi\right\rangle, \\
\left\langle\hat{k}_{i}^{\mathrm{bH}}(z) q_{j}^{\mathrm{A}}\right\rangle & =\left\langle k_{i}^{\mathrm{b}} q_{j}^{\mathrm{A}}\right\rangle+z c_{i}\left\langle k_{j}^{\mathrm{b}} q_{j}^{\mathrm{A}}\right\rangle,
\end{aligned}
$$

\footnotetext{
${ }^{4}$ No such contribution arises for the longitudinal gauge bosons, since the vector $r_{k}$ in the Ward identity (4.13) is proportional to the reference spinors $\eta_{\alpha} q_{k, \dot{\alpha}}$ and does not contribute to $\alpha$.

${ }^{5}$ Here we exclude two-line BCFW shifts, where only one momentum is shifted holomorphically.
} 
while the choice of shift and reference spinors implies that $\left\langle\hat{k}_{i}^{b \mathrm{H}}(z) q_{k}^{\mathrm{H}}\right\rangle$ and $\left\langle\hat{k}_{i}^{b \mathrm{H}}(z) \hat{k}_{j}^{b \mathrm{~A}}\right\rangle$ are not shifted and in particular $\left\langle q_{i}^{\mathrm{H}} \hat{k}_{j}^{b_{\mathrm{A}}}\right\rangle=0$. Therefore the relevant contributions to the holomorphic spinor products in (4.25) are of the form

$$
\left\langle\hat{k}_{i}^{\mathrm{bH}}(z) \cdot\right\rangle^{\alpha}=\left\langle\hat{k}_{i}^{\mathrm{bH}}(z) \hat{k}_{k}^{\mathrm{bH}}(z)\right\rangle^{\alpha_{1}}\left\langle\hat{k}_{i}^{\mathrm{bH}}(z) \chi\right\rangle^{\alpha_{2}}\left\langle\hat{k}_{i}^{\mathrm{bH}}(z) q_{j}^{\mathrm{A}}\right\rangle^{\alpha_{3}} .
$$

Following the reasoning for the Risager-type shift, $\alpha$ can be bound by half the number of the holomorphic spinors contributing to these spinor products. The single anti-holomorphically shifted particle can contribute spinors $k_{j, \alpha}^{\mathrm{A}}$ and $q_{j, \alpha}^{\mathrm{A}}$, where the former drops out of (4.35) while the letter can contribute to $\alpha_{3}$. The number of relevant spinors receives the following contributions:

- The number of holomorphic spinors in $N_{h, b}$, the holomorphically shifted $\hat{u}_{i}^{\mathrm{H}}\left(-\frac{1}{2}\right)$, $\hat{\epsilon}_{i}^{\mathrm{H}}(-)$ and $\hat{\epsilon}_{i}^{\mathrm{H}}(+)$, and the background contributions as in the Risager-type shift.

- The anti-holomorphically shifted polarization vector $\hat{\epsilon}_{j}^{\mathrm{A}}(+)$, since it includes a spinor $q_{j, \alpha}^{\mathrm{A}}$ and therefore contributes to $\alpha_{3}$.

- For $\hat{\epsilon}_{j}^{\mathrm{A}}(-)$, the term $k_{j}^{\mu} A_{\mu}(W, \ldots)$ in the Ward identity (4.16) contains a term involving the reference spinor $q_{j, \alpha}^{\mathrm{A}}$ in the massive case. Similarly, the term involving $r_{k_{j}, \alpha \dot{\alpha}} \propto q_{j, \alpha}^{\mathrm{A}} \eta_{\dot{\alpha}}$ in the Ward identity (4.13) contributes for $\hat{\epsilon}_{j}^{\mathrm{A}}(0)$.

- In the massive case, the spinor $\hat{u}_{j}^{\mathrm{A}}\left(+\frac{1}{2}\right)$ contains the reference spinor $q_{j, \alpha}^{\mathrm{A}}$ and contributes one-half to $\alpha_{3}$. However, either only the holomorphic or the anti-holomorphic part of a Dirac spinor contributes to a given term in the amplitude. Since the possible contribution to $\alpha_{3}$ is smaller than the contribution of the anti-holomorphic component of $\hat{u}_{j}^{\mathrm{A}}\left(+\frac{1}{2}\right)$ to $\beta$ already included in (4.31), the former can be dropped.

Taking all of the contributions to $\alpha$ into account and adding the explicit powers of $z$ due to the application of the Ward identities gives the bound

$$
\begin{aligned}
& \alpha-h_{W(+)}^{\mathrm{H}}-h_{W(-)}^{\mathrm{A}} \\
& \leq \frac{1}{2}\left(\left[N_{h, b}\right]+b_{\psi}+b_{W}+h_{\psi(-)}^{\mathrm{H}}+h_{W(-)}^{\mathrm{H}}-h_{W(+)}^{\mathrm{H}}+h_{W(+)}^{\mathrm{A}}+h_{W(0)}^{\mathrm{A}}-h_{W(-)}^{\mathrm{A}}\right) \\
& =\frac{1}{2}\left(4-h-\min [g]-b_{\phi}-\frac{b_{\psi}}{2}-\sum_{\mathcal{H}} s_{i}+\sum_{\mathcal{A}_{W}} s_{j}-\frac{h_{\psi}^{\mathrm{A}}}{2}+h_{W(0)}^{\mathrm{A}}\right)+\gamma_{D},
\end{aligned}
$$

where the expression for the dimension of the skeleton amplitude (4.6) was used and the spin sums were introduced as discussed above after (4.29). Adding the contribution from the anti-holomorphic spinors (4.31), the bound on the scaling of the amplitude can be written in a form similar to the bound for the Risager shift and an additional contribution depending on the spin of the single anti-holomorphically shifted particle,

$$
\gamma_{\mathrm{BCFW}}^{\mathrm{H}}=\gamma_{N}-\gamma_{D} \leq \frac{1}{2}\left(4-h-\min [g]-b_{\phi}-\frac{b_{\psi}}{2}-\sum_{\mathcal{S}} s_{i}\right)+ \begin{cases}2 s_{j} & W^{+, \mathrm{A}}, \psi^{+, \mathrm{A}} \\ \frac{1}{2}, & W^{0, \mathrm{~A}} \\ s_{j}, & W^{-, \mathrm{A}}, \psi^{-, \mathrm{A}}\end{cases}
$$


For a dominantly anti-holomorphic BCFW-type shift one obtains analogously

$$
\gamma_{\mathrm{BCFW}}^{\mathrm{A}} \leq \frac{1}{2}\left(4-h-\min [g]-b_{\phi}-\frac{b_{\psi}}{2}+\sum_{\mathcal{S}} s_{i}\right)+ \begin{cases}-2 s_{j} & W^{-, \mathrm{A}}, \psi^{-, \mathrm{A}} \\ \frac{1}{2}, & W^{0, \mathrm{~A}} \\ -s_{j}, & W^{+, \mathrm{A}}, \psi^{+, \mathrm{A}}\end{cases}
$$

The corresponding result for holomorphic shift in the massless case [5] is

$$
\gamma_{\mathrm{BCFW}}^{\mathrm{H}, m=0} \leq \frac{1}{2}\left(4-h-\min [g]-\sum_{\mathcal{S}} s_{i}\right)+2 s^{\mathrm{A}} .
$$

The better behaviour for the "good shifts" in the massless case can be understood as follows:

- For $\psi^{-, \mathrm{A}}$ one can argue in the massless case that the change from the holomorphic to the anti-holomorphic shift improves the behaviour by one power of $z$. In the massive case the spinor $\hat{u}_{i}^{\mathrm{H}}\left(-\frac{1}{2}\right)$ also contains a $z$-independent anti-holomorphic component so not all contributions to the amplitude are improved.

- For $W^{-, \mathrm{A}}$ the Ward identity (4.16) gives rise to a contribution involving the reference spinor that does not appear in the massless case.

\subsection{Examples}

We will illustrate the bounds (4.30) and (4.37) for simple examples in order to illuminate differences of the massive and massless cases more concretely. As a first example, consider a mostly holomorphic four-line BCFW-type shift (3.59),

$$
\hat{k}_{i, \alpha}^{b \mathrm{H}}(z)=k_{i, \alpha}^{b}+z c_{i} k_{4, \alpha}^{b}, \quad \hat{k}_{4, \dot{\alpha}}^{b \mathrm{~A}}(z)=k_{4, \dot{\alpha}}^{b}+z d_{4} q_{4, \dot{\alpha}},
$$

for the amplitude

$$
A_{4}\left(\bar{\psi}_{1}^{-, \mathrm{H}}, \phi_{2}^{\mathrm{H}}, \phi_{3}^{\mathrm{H}}, \psi_{4}^{-, \mathrm{A}}\right)=\underbrace{\phi_{2}}_{\psi_{1}}
$$

in Yukawa theory. In the massless case, according to the bound (4.39) the shift is allowed with $\gamma_{\mathrm{BCFW}}^{\mathrm{H}, m=0} \leq-\frac{1}{2}$ whereas in the massive case the estimate (4.37) gives $\gamma_{\mathrm{BCFW}}^{\mathrm{H}} \leq 0$. For massless fermions, due to helicity selection rules, the only contribution to the amplitude arises from the diagram with a triple-scalar vertex,

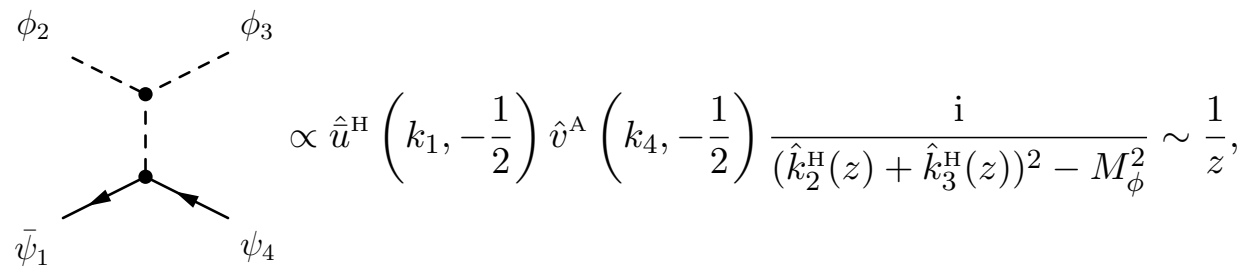


since the shift drops out of the product of the Dirac spinors (3.30) and (3.32),

$$
\hat{\bar{u}}^{\mathrm{H}}\left(k_{1},-\frac{1}{2}\right) \hat{v}^{\mathrm{A}}\left(k_{4},-\frac{1}{2}\right)=\left\langle\hat{k}_{1}^{\mathrm{bH}}(z) k_{4}^{b}\right\rangle=\left\langle k_{1}^{b} k_{4}^{b}\right\rangle \sim z^{0} .
$$

In the massive case, there is a non-vanishing contribution from the fermion exchange diagrams, which include a $z$-dependent contribution in the numerator, for example

$$
\underbrace{\sim}_{z \rightarrow \infty} \frac{\left\langle k_{1}^{\mathrm{H}}(z)\left|\hat{k}_{3}^{\mathrm{H}}(z)\right| q_{4}\right] m_{\psi}}{\left(\hat{k}_{3}^{\mathrm{H}}(z)+\hat{k}_{4}^{\mathrm{A}}(z)\right)^{2}-m_{\psi}^{2}} \sim \frac{z\left(c_{1}\left\langle k_{4}^{\mathrm{b}} k_{3}^{\mathrm{b}}\right\rangle+c_{3}\left\langle k_{1}^{\mathrm{b}} k_{4}^{\mathrm{b}}\right\rangle\right)\left[k_{3}^{\mathrm{b}} q_{4}\right] m_{\psi}}{\left(\hat{k}_{3}^{\mathrm{H}}(z)+\hat{k}_{4}^{\mathrm{A}}(z)\right)^{2}-m_{\psi}^{2}} \sim z^{0},
$$

so that the massive bound (4.37) is saturated. This illustrates how mass-suppressed contributions can give rise to a worse large- $z$ behaviour than in the massless case.

In this example, this complication is not relevant in praxis since the amplitude is constructible using an anti-holomorphic Risager shift of all lines,

$$
\hat{k}_{i, \dot{\alpha}}^{\mathrm{bA}}(z)=k_{i, \dot{\alpha}}^{b}+z d_{i} \eta_{\dot{\alpha}},
$$

with the reference spinors $q_{i, \dot{\alpha}}=\eta_{\dot{\alpha}}$. In agreement with the anti-holomorphic version of (4.30) one obtains for the diagram with a fermion propagator

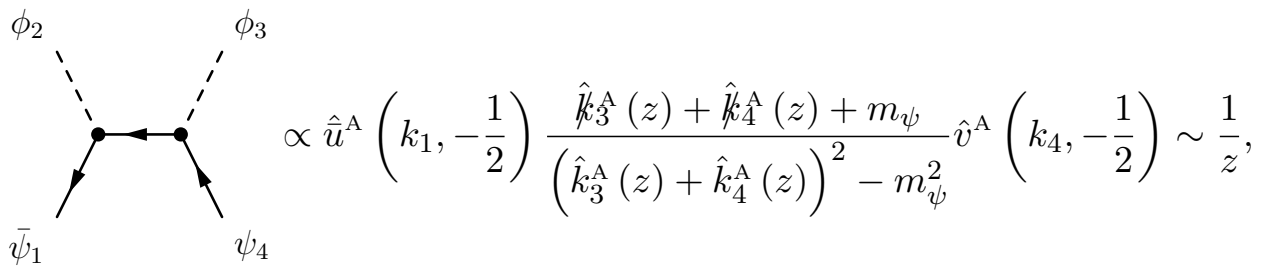

since in this case the choice of reference spinors ensures that the shift drops out in the numerator, $\left.\left.\hat{\not k}_{3}^{\mathrm{A}}(z) \mid q_{4}\right]=\not k_{3} \mid \eta\right]$, and similarly for spinor chains involving $\left[q_{1} \mid\right.$. This example also illustrates that the large- $z$ behaviour of amplitudes with fixed spin quantum numbers generally depends on the choice of the spin axes, in contrast to the massless case where the reference spinors are unphysical auxiliary quantities.

As an example for the large- $z$ behaviour of amplitudes with massive vector bosons for the BCFW-type shift (4.40), consider an amplitude with two vector bosons and two scalars in the Abelian Higgs model in unitary gauge, ${ }^{6}$

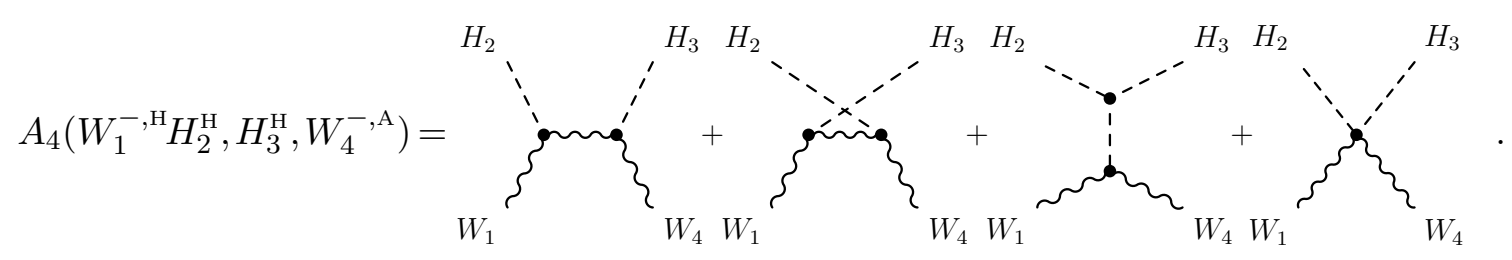

\footnotetext{
${ }^{6}$ In the 't Hooft-Feynman gauge also diagrams with Goldstone-boson exchange contribute, while the $W W H$ vertex vanishes in the massless limit. This is, however, irrelevant to the estimate of the large- $z$ behaviour below.
} 
In the massless case the shift is allowed according to the bound $\gamma_{\mathrm{BCFW}}^{\mathrm{H}, m=0} \leq-1$, whereas in the massive case the weaker estimate $\gamma_{\mathrm{BCFW}}^{\mathrm{H}} \leq 0$ is obtained from (4.37). This difference can be traced to the different role of the reference spinors $q_{i}$ in the massless and massive cases. The diagram with the four-point vertex is proportional to

$$
\hat{\epsilon}^{\mathrm{H}}\left(k_{1},-\right) \cdot \hat{\epsilon}^{\mathrm{A}}\left(k_{4},-\right)=\frac{\left\langle k_{1}^{\mathrm{b}} k_{4}^{\mathrm{b}}\right\rangle\left[q_{1} q_{4}\right]}{\left[k_{1}^{\mathrm{b}} q_{1}\right]\left[k_{4}^{\mathrm{b}} \eta\right]},
$$

which can be made to vanish in the massless case by choosing equal reference spinors, without affecting the result for the amplitude. In the massive case the choice of reference spinors determines the spin axis and therefore the physical result. For the choice of reference spinors made in section 5, the scalar product does not vanish so the bound (4.37) is saturated. The same conclusion is reached using the Ward identity (4.16),

$$
A_{4}\left(W_{1}^{-, \mathrm{H}} H_{2}^{\mathrm{H}}, H_{3}^{\mathrm{H}}, W_{4}^{-, \mathrm{A}}\right) \propto \frac{1}{z}\left(m_{W_{4}} A_{4}\left(W_{1}^{-, \mathrm{H}} H_{2}^{\mathrm{H}}, H_{3}^{\mathrm{H}}, \phi_{4}^{\mathrm{A}}\right)-k_{4, \mu} A_{4}^{\mu}\left(W_{1}^{-, \mathrm{H}} H_{2}^{\mathrm{H}}, H_{3}^{\mathrm{H}}, W_{4}^{\mathrm{A}}\right)\right) .
$$

Focusing on the second term, the diagram with the four-point vertex is proportional to

$$
\hat{\epsilon}^{\mathrm{H}}\left(k_{1},-\right) \cdot k_{4}=\frac{\left\langle k_{1}^{b} k_{4}^{b}\right\rangle\left[k_{4}^{b} q_{1}\right]}{\left[k_{1}^{b} q_{1}\right]}+\frac{m_{W_{4}}^{2}}{2\left(q_{4} \cdot k_{4}\right)} \frac{\left\langle\hat{k}_{1}^{b}(z) q_{4}\right\rangle\left[q_{4} q_{1}\right]}{\left[k_{1}^{b} q_{1}\right]} \propto z,
$$

so again equal reference spinors are required to obtain a valid shift. It is obvious that the problematic term is absent in the massless case. As for the example in Yukawa theory, in this case a valid Risager shift is available.

\section{On-shell constructible amplitudes}

In this section we establish that the following shifts are sufficient to construct all amplitudes in SBGTs with at least five legs (or six legs for all-fermionic amplitudes):

- Three-line and in some cases two-line shifts are sufficient for the following amplitudes:

- All amplitudes with at least two transverse vector bosons.

- Amplitudes with scalars and at least two vector bosons, at least one of which is transverse.

- All amplitudes that contain only fermions; fermions and vector bosons; fermions and SM-like Higgs bosons; or generic scalars and at least two fermion pairs.

- Five-line shifts are required for amplitudes with only scalars.

- Four-line shifts are sufficient for all other cases.

Here "SM-like" Higgs bosons are defined as scalars that couple to vector bosons through vertices of type $H W W$ but not through $H H W$ vertices. Furthermore, three-line shifts are sufficient for amplitudes of scalars charged under an unbroken U(1) symmetry [5], which is, however, not present for the SM Higgs boson in the broken phase. The above conditions 
apply when all legs are massive. For at least two massless vector bosons or fermions, the familiar conditions for massless BCFW shifts $[2,23,24,40]$ can be used. We do not consider the case of shifts with one massless particle explicitly; see [23] for a discussion of shifts with massive and massless quarks and gluons. Compared to the results for an unbroken gauge theory with fermionic and scalar matter fields [5], the new feature are four-line shifts for longitudinal gauge bosons, which arise by exploiting little-group transformations.

The above statements are derived in the remainder of this section. In the study of the $z \rightarrow \infty$ behaviour we make use of the ability to perform different types of shifts for different spin states of the external particles. This requires a choice of spin axes for the shifted particles so that all required shifts are feasible. Such a choice is introduced in section 5.1. The conditions for allowed shifts for this setup are summarized in section 5.2 while the minimal shifts required for the construction of different classes of amplitudes are investigated in section 5.3.

\subsection{Choice of reference spinors for all spin configurations}

To define the spin axes for all particles in a way that allows to perform different types of shifts depending on the spins of the particles, two "reference particles" will be singled out. For definiteness, these particles will be assigned the momenta $k_{1}$ and $k_{n}$. Since the choice of a spin axis is not necessary for purely scalar amplitudes, in all relevant cases it is possible to choose at least one particle with spin as reference particle. As in (3.40) the reference momenta can be expressed in terms of two light-like vectors $l_{1 / n}$ according to

$$
k_{1}=l_{1}+\alpha_{n} l_{n}, \quad k_{n}=\alpha_{1} l_{1}+l_{n} .
$$

The reference spinors for the two selected legs are chosen as

$$
\begin{aligned}
& q_{1, \alpha}=l_{n, \alpha}, \quad q_{1, \dot{\alpha}}=l_{n, \dot{\alpha}}, \\
& q_{n, \alpha}=l_{1, \alpha}, \quad q_{n, \dot{\alpha}}=l_{1, \dot{\alpha}},
\end{aligned}
$$

while those for all other legs are taken as

$$
q_{i, \alpha}=l_{n, \alpha}, \quad q_{i, \dot{\alpha}}=l_{1, \dot{\alpha}}
$$

A similar construction was used in [23].

Provided the amplitudes for all spin states can be computed recursively with this choice of spin axes, the amplitudes for arbitrary spin axes follow from little-group transformations. It is even sufficient to keep the spin quantum number of one particle fixed, for instance by taking $s_{n}$ as negative. This information allows to reconstruct the amplitudes for arbitrary spin axes of the remaining legs using the little-group transformations (2.27) and (2.39),

$$
A\left(\Phi_{1}^{s_{1}^{\prime}}, \ldots, \Phi_{n-1}^{s_{n-1}^{\prime}}, \Phi_{n}^{-}\right)=\sum_{s_{1}} \cdots \sum_{s_{n-1}} \mathcal{R}_{s_{1}^{\prime}, s_{1}}^{\left(S_{1}\right)} \ldots \mathcal{R}_{s_{n-1}^{\prime}, s_{n-1}}^{\left(S_{n-1}\right)} A\left(\Phi_{1}^{s_{1}}, \ldots, \Phi_{n-1}^{s_{n-1}}, \Phi_{n}^{-}\right) .
$$

Since now the spin axes for all particles are independent, the operator $J_{k_{n}}^{+}(2.16)$ can be used to raise the spin of the last particle. 


\subsection{Explicit form of shifts}

The choice of reference spinors of section 5.1 allows to perform all of the types of shifts constructed in section 3 . We summarize the explicit form and the conditions for the shifted legs in order to obtain an allowed shift.

Two-line BCFW-type shift. The choice of reference spinors (5.2) and (5.3) allows to shift the two reference particles by a two-line BCFW shift (3.44),

$$
\begin{array}{ll}
\hat{k}_{1, \alpha}^{b, \mathrm{H}}(z)=l_{1, \alpha}+z l_{n, \alpha}, & \hat{k}_{1, \dot{\alpha}}^{b, \mathrm{H}}(z)=l_{1, \dot{\alpha}} \\
\hat{k}_{n, \alpha}^{b, \mathrm{~A}}(z)=l_{n, \alpha}, & \hat{k}_{n, \dot{\alpha}}^{b \mathrm{~A}}(z)=l_{n, \dot{\alpha}}-z l_{1, \dot{\alpha}} .
\end{array}
$$

For the internal lines in the recursion relation (3.11), the reference spinors $q_{\mathcal{F}, \dot{\alpha}}=\eta_{\dot{\alpha}}=l_{1, \dot{\alpha}}$ and $q_{\mathcal{F}, \alpha}=\eta_{\alpha}=l_{n, \alpha}$ are the same as for the external legs (5.4). A valid recursion relation is obtained from the bound (4.24) if

$$
\gamma \leq 1-s_{1}+s_{n}<0 .
$$

This allows to shift the following combinations of particles,

$$
W_{1}^{+, \mathrm{H}} W_{n}^{-, \mathrm{A}}, \quad \psi_{1}^{+, \mathrm{H}} W_{n}^{-, \mathrm{A}}, \quad W_{1}^{+, \mathrm{H}} \psi_{n}^{-, \mathrm{A}}, \quad \psi_{1}^{+, \mathrm{H}} \psi_{n}^{-, \mathrm{A}} .
$$

Two fermions may only be shifted if they belong to different fermion lines [23] since in this case the skeleton amplitude necessarily includes two fermionic background insertions so that the condition (5.7) improves to

$$
\gamma \leq-s_{1}+s_{n}<0
$$

For amplitudes with massless particles not all allowed shifts are found by this simple powercounting analysis; in particular shifts of particles with identical helicities are possible [2]. We argue at the end of section 5.3 that similar improvements are not expected for shifts of massive particles.

Holomorphic Risager-type shift. An $h$-line shift, which may include the reference momentum $k_{1}$ but not $k_{n}$, is possible with the shift spinor $\eta_{\alpha}=l_{n, \alpha}$ and the shifted momenta

$$
\hat{k}_{i, \alpha}^{b, \mathrm{H}}(z)=k_{i, \alpha}^{\mathrm{b}}+z c_{i} l_{n, \alpha}, \quad \hat{k}_{i, \dot{\alpha}}^{b, \mathrm{H}}(z)=k_{i, \dot{\alpha}}^{b} .
$$

Therefore $h+1$-point functions can be constructed with an $h$-line Risager shift for the above choice of spin axes. ${ }^{7}$ The reference spinors for the internal line in the recursion (3.11) are fixed according to (3.50), which implies that the holomorphic reference spinor $q_{\mathcal{F}, \alpha}=\eta_{\alpha}=l_{n, \alpha}$ in the subamplitudes is the same as for the full amplitude. According to the bound (4.30), a valid $h$-line recursion relation for dimensionless coupling constants is obtained if the spin projections of the shifted legs satisfy

$$
\sum_{\mathcal{S}} s_{i}>4-h
$$

\footnotetext{
${ }^{7}$ Since a choice of spin axes is not necessary for all-scalar amplitudes, the five-scalar amplitude can be constructed from a five-line shift.
} 
Anti-holomorphic Risager-type shift. In an $h$-line shift with shift spinor $\eta_{\dot{\alpha}}=l_{1, \dot{\alpha}}$ all legs including the reference momentum $k_{n}$, but not $k_{1}$, can be shifted as

$$
\hat{k}_{j, \alpha}^{b, \mathrm{~A}}(z)=k_{j, \alpha}^{\mathrm{b}}, \quad \hat{k}_{j, \dot{\alpha}}^{b, \mathrm{~A}}(z)=k_{j, \dot{\alpha}}^{b}+z d_{j} l_{1 \dot{\alpha}} .
$$

The subamplitudes inherit the anti-holomorphic reference spinor, $q_{\mathcal{F}, \dot{\alpha}}=\eta_{\dot{\alpha}}=l_{1, \dot{\alpha}}$. A valid recursion relation is obtained for

$$
\sum_{\mathcal{S}} s_{j}<-(4-h)
$$

Mostly holomorphic BCFW-type shift. An holomorphic shift is applied to $h-1$ momenta (which may include $k_{1}$ ) while the reference momentum $k_{n}$ is shifted antiholomorphically,

$$
\begin{aligned}
\hat{k}_{i, \alpha}^{\mathrm{b}, \mathrm{H}}(z) & =k_{i, \alpha}^{\mathrm{b}}+z c_{i} l_{n, \alpha}, & \hat{k}_{i, \dot{\alpha}}^{\mathrm{b}, \mathrm{H}}(z) & =k_{i, \dot{\alpha}}^{\mathrm{b}}, \\
\hat{k}_{n, \alpha}^{b, \mathrm{~A}}(z) & =l_{n, \alpha}, & \hat{k}_{n, \dot{\alpha}}^{\mathrm{A}}(z) & =l_{n, \dot{\alpha}}+z d_{n} l_{1, \dot{\alpha}} .
\end{aligned}
$$

As for the holomorphic Risager shift, the subamplitudes have the same common holomorphic reference spinor $q_{\mathcal{F}, \alpha}=\eta_{\alpha}=l_{n, \alpha}$ according to (3.60). If leg $n$ has negative spin projection, a valid recursion relation is obtained from (4.37) for

$$
\sum_{\mathcal{S}} s_{i}-2 s_{n}^{\mathrm{A}}=\sum_{\mathcal{H}} s_{i}+\left|s_{n}^{\mathrm{A}}\right|>4-h .
$$

Mostly anti-holomorphic BCFW-type shift. Here the reference momentum $k_{1}$ is shifted holomorphically and $h-1$ momenta (which may include $k_{n}$ ) are shifted antiholomorphically,

$$
\begin{array}{ll}
\hat{k}_{1, \alpha}^{b, \mathrm{H}}(z)=l_{1, \alpha}+z c_{1} l_{n, \alpha}, & \hat{k}_{1, \dot{\alpha}}^{\mathrm{b}, \mathrm{H}}(z)=l_{1, \dot{\alpha}}, \\
\hat{k}_{j, \alpha}^{\mathrm{b}}(z)=k_{j, \alpha}^{\mathrm{b}}, & \hat{k}_{j, \dot{\alpha}}^{\mathrm{bA}}(z)=k_{j, \dot{\alpha}}^{\mathrm{b}}+z d_{j} l_{1, \dot{\alpha}} .
\end{array}
$$

The subamplitudes have the same common anti-holomorphic reference spinor $q_{\mathcal{F}, \dot{\alpha}}=\eta_{\dot{\alpha}}=$ $l_{1, \dot{\alpha}}$. If leg one has positive spin projection, a valid recursion relation is obtained for

$$
\sum_{\mathcal{S}} s_{j}-2 s_{1}^{\mathrm{H}}=\sum_{\mathcal{A}} s_{j}-\left|s_{1}^{\mathrm{H}}\right|<-(4-h) .
$$

\subsection{Minimal required shifts}

It is now possible to identify the minimal number of shifted legs necessary to construct a given amplitude and verify the claims made in the beginning of this section. Recall that little-group transformations allow to reconstruct amplitudes with general spin quantum numbers from amplitudes where the reference particle $n$ has spin $-\frac{1}{2}$ or -1 , provided the amplitudes for arbitrary spin configurations of the remaining particles are known for a fixed choice of reference legs 1 and $n$. 


\subsubsection{Five-line constructible amplitudes}

All amplitudes are constructible either from holomorphic or anti-holomorphic five-line Risager shifts since the condition $\left|\sum_{\mathcal{S}} s_{i}\right| \geq 0$ is obviously always satisfied. This agrees with the conclusion from the massless case [5].

\subsubsection{Four-line constructible amplitudes}

The following four-line shifts are possible:

- Holomorphic (anti-holomorphic) Risager shifts for $\sum_{\mathcal{H}} s_{i}>0\left(\sum_{\mathcal{A}} s_{j}<0\right)$.

- Mostly holomorphic BCFW-type shifts provided the holomorphically shifted particles satisfy $\sum_{\mathcal{H}} s_{i}>-\left|s_{n}\right|$ for $s_{n}<0$.

- Mostly anti-holomorphic BCFW-type shifts provided the anti-holomorphically shifted particles satisfy $\sum_{\mathcal{A}} s_{j}<s_{1}$ for $s_{1}>0$.

Therefore all amplitudes are four-line Risager constructible unless $\sum_{\mathcal{S}} s_{i}=0$. In this case, amplitudes with at least one fermion pair or vector boson can be constructed with a BCFW-type shift with a particle of negative spin projection as reference leg $n$. The only amplitudes that are not four-line constructible are therefore amplitudes with only scalars. ${ }^{8}$

\subsubsection{Three-line constructible amplitudes}

The following three-line shifts are possible:

- Holomorphic (anti-holomorphic) Risager shifts for $\sum_{\mathcal{H}} s_{i}>1\left(\sum_{\mathcal{A}} s_{j}<-1\right)$.

- Mostly holomorphic BCFW-type shifts provided the holomorphically shifted particles satisfy $\sum_{\mathcal{H}} s_{i}>1-\left|s_{n}\right|$ for $s_{n}<0$

- Mostly anti-holomorphic BCFW-type shifts provided the anti-holomorphically shifted particles satisfy $\sum_{\mathcal{A}} s_{j}<s_{1}-1$ for $s_{1}>0$.

We now determine all amplitudes that can be constructed using three-line shifts, ensuring that all spin configurations can be computed for a fixed choice of reference particles. In some cases, also the two-line shifts (5.8) can be used.

Vector-boson amplitudes. Two vector bosons $W_{1}^{s_{1}}$ and $W_{n}^{-}$are selected as reference particles. A two-line BCFW shift is possible for the spin configuration $W_{1}^{+, \mathrm{H}} W_{n}^{-, \mathrm{A}}$. For other spin projections of $W_{1}$, anti-holomorphic Risager shifts $W_{i}^{s_{i}, \mathrm{~A}} W_{j}^{s_{j}, \mathrm{~A}} W_{n}^{-, \mathrm{A}}$ can be applied if the amplitude contains two further vector bosons with $s_{i}+s_{j} \leq-1$ while mostly holomorphic BCFW shifts $W_{i}^{s_{i}, \mathrm{H}} W_{j}^{s_{j}, \mathrm{H}} W_{n}^{-, \mathrm{A}}$ are possible for $s_{i}+s_{j} \geq 1$. These shifts cover all amplitudes with at least five vector bosons apart from those where all vector bosons besides the two reference legs are longitudinal, where four-line shifts can be applied as discussed above.

\footnotetext{
${ }^{8}$ Amplitudes with only longitudinal vector bosons can be obtained from the allowed four-line BCFW shift $W^{0, \mathrm{H}} W^{0, \mathrm{H}} W^{0, \mathrm{H}} W_{n}^{-, \text {A }}$ using the little group raising operator on the reference leg with negative spin.
} 
Amplitudes with scalars and vector bosons. A scalar $\phi_{1}$ and a vector boson $W_{n}^{-}$can be selected as reference particles. For amplitudes with at least one additional transverse vector boson $W_{i}$, either a mostly-holomorphic BCFW shift $\phi_{1}^{\mathrm{H}} W_{i}^{+, \mathrm{H}} W_{n}^{-, \mathrm{A}}$ is possible or one can select a further particle $\Phi_{j} \in\left\{W_{j}^{-}, W_{j}^{0}, \phi_{j}\right\}$ so that an anti-holomorphic Risager shift $W_{i}^{-, \mathrm{A}} \Phi_{j}^{\mathrm{A}} W_{n}^{-, \mathrm{A}}$ can be performed. This covers all amplitudes with scalars and at least two vector bosons, unless all vector bosons besides $W_{n}^{-}$are longitudinal where again a four-line shift is required.

For amplitudes with scalars and only one vector boson, neither the condition $\sum_{\mathcal{A}} s_{j}<-1$ for a Risager shift or $\sum_{\mathcal{H}} s_{i}>0$ for a mostly holomorphic BCFW shift can be satisfied. However, such amplitudes do not appear for SM-like Higgs bosons, which only couple to pairs of vector bosons.

Amplitudes with scalars and fermions. A scalar $\phi_{1}$ and a fermion $\psi_{n}^{-}$can be chosen as reference particles. For amplitudes with at least two fermion pairs there must be two additional fermions with equal spin quantum number so that a mostly-holomorphic BCFW shift $\psi_{i}^{+, \mathrm{H}} \psi_{j}^{+, \mathrm{H}} \psi_{n}^{-, \mathrm{A}}$ or an anti-holomorphic Risager shift $\psi_{i}^{-, \mathrm{A}} \psi_{j}^{-, \mathrm{A}} \psi_{n}^{-, \mathrm{A}}$ are possible.

For amplitudes with only one fermion pair, the conditions for a three-line shift cannot be satisfied for generic scalar particles. However, the situation improves for SM-like Higgs bosons without $H H W$ couplings. In this case there are no internal vector-boson lines and at least one scalar background insertion must appear in the skeleton amplitudes for the shifts $\phi^{\mathrm{H}} \psi^{+, \mathrm{H}} \psi_{n}^{-, \mathrm{A}}$ and $\phi^{\mathrm{A}} \psi^{-, \mathrm{A}} \psi_{n}^{-, \mathrm{A}}$. This improves the scaling, for example in case of the BCFW-type shift (4.37),

$$
\gamma_{\mathrm{BCFW}}^{\mathrm{H}} \leq \frac{1}{2}\left(1-b_{\phi}\right)-\left|s_{n}^{\mathrm{A}}\right|=-\frac{1}{2},
$$

and similarly for the Risager shift (4.30). Therefore, all amplitudes with SM-like Higgs bosons and one fermion pair are three-line constructible. For the case of four-point amplitudes, this can be checked in the example given in section 4.5 .

Amplitudes with a fermion pair and vector bosons. A fermion $\psi_{1}^{s_{1}}$ and a vector boson $W_{n}^{-}$can be taken as reference particles. A two-line BCFW shift can be performed for the spin configuration $\psi_{1}^{+, \mathrm{H}} W_{n}^{-, \mathrm{A}}$. Otherwise, a Risager shift $\Phi_{i}^{s_{i} \mathrm{~A}} \Phi_{j}^{s_{j}, \mathrm{~A}} W_{n}^{-, \mathrm{A}}$ or a BCFW-type shift $\Phi_{i}^{s_{i}, \mathrm{H}} \Phi_{j}^{s_{j} \mathrm{H}} W_{n}^{-, \mathrm{A}}$ can be performed if the amplitude contains two additional particles with $s_{i}+s_{j}<0$ or $s_{i}+s_{j}>0$, respectively. Since the amplitude must contain a second fermion with $s= \pm \frac{1}{2}$, one of these conditions can always be fulfilled for amplitudes with arbitrary remaining particle content.

Amplitudes with only fermions. We consider all-fermionic amplitudes with at least six legs and assume every fermion can be uniquely assigned to one fermion line for all Feynman diagrams contributing to the amplitude. ${ }^{9}$ Then two fermions from different fermion lines can be chosen as reference particles $\psi_{1}^{s_{1}}$ and $\psi_{n}^{-}$. If reference particle one has positive spin, a two-line BCFW shift $\psi_{1}^{+, \mathrm{H}} \psi_{n}^{-, \mathrm{A}}$ is possible. For negative spin projection of

\footnotetext{
${ }^{9}$ It is always possible to construct partial amplitudes with this property by assigning the fermions pairs to different, possibly artificial, generations and assuming flavour-mixing matrices to be diagonal.
} 
leg one, it is always possible to perform a mostly-holomorphic BCFW shift $\psi_{i}^{+, \mathrm{H}} \psi_{j}^{+, \mathrm{H}} \psi_{n}^{-, \mathrm{A}}$ or an anti-holomorphic Risager shift $\psi_{i}^{-, \mathrm{A}} \psi_{j}^{-, \mathrm{A}} \psi_{n}^{-, \mathrm{A}}$ since two of the remaining (at least four) fermions must have the same spin quantum number. This conclusion agrees with [23], however, here we do not require the additional condition that the reference particle $\psi_{1}$ and the three shifted legs all belong to different fermion lines.

Therefore we have verified all cases of three-line constructible amplitudes given in the beginning of section 5 .

Further improvements? As mentioned above, for massless particles also two-line BCFW shifts of legs with equal helicity are allowed, although not obvious from simple power-counting. This may be proven for instance using an auxiliary three-line recursion $[18,23]$ or a background field analysis [24]. While we have not performed a comprehensive analysis, such improvements do not appear to be possible for massive particles. For massive quarks, it is known that BCFW shifts of two legs with the same spin are not allowed [23]. As a starting point of the inductive proof using auxiliary shifts, three-point amplitudes involving the two shifted legs must vanish for $z \rightarrow \infty$ [23]. For a two-line shift of two massive vector bosons $W_{1}^{-, \mathrm{H}} W_{n}^{-, \mathrm{A}}$ and the choice of reference spinors as in section 5.1, this induction assumption is violated since three-point functions for an unshifted vector boson $W_{i}^{-}$and the two shifted vector bosons do not vanish for $z \rightarrow \infty$,

$$
A_{3}\left(\hat{W}_{1}^{-, \mathrm{H}}, W_{i}^{-}, \hat{W}_{n}^{-, \mathrm{A}}\right) \propto\left(\hat{\epsilon}^{\mathrm{H}}\left(k_{1},-\right) \cdot \hat{\epsilon}^{\mathrm{A}}\left(k_{n},-\right)\right)\left(\epsilon\left(k_{i},-\right) \cdot k_{n}^{\mathrm{A}}(z)\right) \sim z^{0},
$$

since $\hat{\epsilon}^{\mathrm{H}}\left(k_{1},-\right) \cdot \hat{\epsilon}^{\mathrm{A}}\left(k_{n},-\right) \neq 0$ as in (4.47). This is another example for the dependence of the large- $z$ behaviour on the choice of spin axis discussed in section 4.5.

\section{Application to selection rules}

Selection rules for helicity amplitudes of massless particles with arbitrary multiplicities are well known, see e.g. [41, 42] for reviews. In particular, gluonic amplitudes vanish if all gluons have the same helicity or only one has a different helicity. The first nonvanishing amplitudes are the so-called maximally helicity violating (MHV) amplitudes, which have a very simple all-multiplicity expression. The corresponding selection rules for amplitudes with massive particles are less restrictive and depend on the choice of spin axes. Selection rules for all-multiplicity amplitudes with massive vector bosons have been derived from supersymmetric identities [8, 43] or using diagrammatic arguments [26]. Here we provide an alternative derivation of some of these results as an application of on-shell recursion relations for amplitudes with massive vector bosons. We furthermore discuss the role played by the choice of spin axes.

For the derivation of the selection rules, it is useful to deviate slightly from the choice of spin axes of section 5.1 and choose the reference spinors of all remaining particles in terms of the momentum and spin axis of one reference particle $n$ :

$$
q_{i, \alpha}=k_{n, \alpha}^{b}, \quad q_{i, \dot{\alpha}}=q_{n, \dot{\alpha}} .
$$


Relations with an exchanged role of positive and negative spin projections can be derived for reference spinors $q_{i, \alpha}=q_{n, \alpha}$ and $q_{i, \dot{\alpha}}=k_{n, \dot{\alpha}}^{b}$. On-shell recursion relations can be used to show that amplitudes with only vector bosons with negative spin projection vanish for the choice (6.1), as well as those with one additional scalar or longitudinal vector boson,

$$
\begin{aligned}
A\left(W_{1}^{-}, W_{2}^{-}, \ldots, W_{n}^{-}\right) & =0, \\
A\left(W_{1}^{0}, W_{2}^{-}, \ldots, W_{n}^{-}\right) & =0, \\
A\left(\phi_{1}, W_{2}^{-}, \ldots, W_{n}^{-}\right) & =0 .
\end{aligned}
$$

Furthermore, amplitudes where the reference particle has negative spin projection vanish if all other vector bosons have positive spin projection, or contain one scalar or longitudinal vector boson,

$$
\begin{aligned}
A\left(W_{1}^{+}, W_{2}^{+}, \ldots, W_{n}^{-}\right) & =0, \\
A\left(W_{1}^{0}, W_{2}^{+}, \ldots, W_{n}^{-}\right) & =0, \\
A\left(\phi_{1}, W_{2}^{+}, \ldots, W_{n}^{-}\right) & =0 .
\end{aligned}
$$

These identities are derived in section 6.1. In section 6.2, similar identities are derived for amplitudes with a fermion pair and vector bosons where all spin projections are negative,

$$
A\left(\bar{\psi}_{1}^{-}, \psi_{2}^{-}, W_{3}^{-}, \ldots, W_{n}^{-}\right)=0,
$$

or all positive with the exception of the reference particle,

$$
\begin{aligned}
A\left(\bar{\psi}_{1}^{+}, \psi_{2}^{+}, W_{3}^{+}, \ldots, \ldots W_{n}^{-}\right) & =0, \\
A\left(\bar{\psi}_{1}^{+}, W_{2}^{+}, \ldots \psi_{n}^{-}\right) & =0 .
\end{aligned}
$$

All amplitudes appearing in these selection rules vanish in the massless limit. For the massive case, however, the selection rules are valid only for the spin axes defined by the choice of reference spinors (6.1). These results are compatible with relations valid in massive supersymmetric theories for the same choice $q_{i, \alpha}=k_{n, \alpha}^{b}$ of holomorphic reference spinors [8]. In section 6.3, little-group transformations are used to transform these selection rules into a frame with a common spin axis for all particles, as used in [26]. In this frame, the selection rules (6.2) and (6.4) continue to hold exactly, while the amplitudes appearing in the other selection rules are mass-suppressed in the high-energy limit.

\subsection{Selection rules for bosonic amplitudes}

For the set of amplitudes (6.2) it is possible to perform a three-line anti-holomorphic Risager shift $W_{l}^{-, \mathrm{A}} W_{m}^{-, \mathrm{A}} W_{n}^{-, \mathrm{A}}$ with arbitrary shift spinor $\eta_{\dot{\alpha}}$ so that the amplitudes satisfy the recursion

$$
\begin{aligned}
& A\left(\Phi_{1}, \ldots W_{l}^{-}, \ldots, W_{m}^{-}, \ldots, W_{n}^{-}\right) \\
& =\sum_{\mathcal{F}, s} A_{\mathcal{F}}\left(\Phi_{1}, \ldots \hat{W}_{l}^{-, \mathrm{A}} \ldots \hat{\Phi}_{\mathcal{F}}^{s}\right) \frac{\mathrm{i}}{K_{\mathcal{F}}^{2}-M_{\mathcal{F}}^{2}} A_{\mathcal{F}^{\prime}}\left(\hat{\Phi}_{\mathcal{F}^{\prime}}^{-s}, \ldots, \hat{W}_{m}^{-, \mathrm{A}} \ldots \hat{W}_{n}^{-, \mathrm{A}} \ldots\right)+\ldots,
\end{aligned}
$$


where $\Phi_{1} \in\left\{W^{-}, W^{0}, \phi\right\}$ and $\Phi_{\mathcal{F}}^{s} \in\left\{W^{s}, \phi\right\}$. The dots indicate similar contributions with a different distribution of the shifted legs over the factorized subamplitudes.

For the anti-holomorphic Risager shift, all scalar products of polarization vectors with negative spin projection vanish for all external and internal lines,

$$
\epsilon\left(k_{i},-\right) \cdot \epsilon\left(k_{j},-\right)=0,
$$

since all external and internal lines are defined using the same anti-holomorphic reference spinor, $q_{\mathcal{F}, \dot{\alpha}}=q_{i, \dot{\alpha}}=q_{n, \dot{\alpha}}$. This implies the selection rules for three-point vertices,

$$
A_{3}\left(\Phi_{i}, W_{j}^{-}, W_{k}^{-}\right)=0
$$

where all particles may be shifted or unshifted, as well as external or internal. The result for $\Phi_{i}=\left\{W_{i}^{-}, \phi_{i}\right\}$ follows trivially since all terms in the vertex involve a scalar product of two polarization vectors. For the longitudinal polarization $\Phi_{i}=W_{i}^{0}$, the selection rule follows from the Ward identity (4.11) since the choice of reference spinors (6.1) implies the identity

$$
\epsilon\left(k_{j},-\right) \cdot r_{i}=0
$$

for all internal or external legs, where the remainder vector $r_{\mu} \propto q_{\mu}$ is defined in (4.14).

The recursion relation (6.7) allows to construct the four-point amplitudes from threepoint amplitudes as input. The selection rules for the three point functions imply that all possible combinations of three-point building blocks appearing in the numerator for the different factorization channels vanish, i.e.

$$
\sum_{s} A_{3}\left(\Phi_{1}, \hat{W}_{l}^{-, \mathrm{A}}, \hat{\Phi}_{\mathcal{F}}^{s}\right) A_{3}\left(\hat{\Phi}_{\mathcal{F}^{\prime}}^{-s}, \hat{W}_{m}^{-, \mathrm{A}}, \hat{W}_{n}^{-, \mathrm{A}}\right)=0
$$

This verifies the selection rules (6.2) for four-point functions. By induction, the argument generalizes to general multiplicities due to the structure of the recursion relation (6.7).

For the amplitudes (6.3) it is possible to perform a mostly-holomorphic BCFW threeline shift (3.59) $W_{l}^{+, \mathrm{H}} W_{m}^{+, \mathrm{H}} W_{n}^{-, \mathrm{A}}$, which leads to a recursion relation of a similar form as (6.7) up to the different spin labels. Internal lines in the recursion relations are defined by the same holomorphic reference spinor $q_{\mathcal{F}, \alpha}=q_{i, \alpha}=k_{n, \alpha}^{b}$ as all external line apart from the reference leg $n$. Therefore the polarization vectors with positive spin projection share the same reference spinor for internal and external lines. This choice implies that the scalar products

$$
\epsilon\left(k_{i},+\right) \cdot \epsilon\left(k_{j},+\right)=0, \quad \epsilon\left(k_{i}, \pm\right) \cdot \hat{\epsilon}^{\mathrm{A}}\left(k_{n},-\right)=0,
$$

vanish where $i$ and $j$ denote arbitrary vector bosons, including the reference leg $n$. This implies the selection rules

$$
A_{3}\left(\Phi_{i}, W_{j}^{+}, W_{k}^{+}\right)=0
$$

with $\Phi_{i}=\left\{W_{i}^{+}, W_{i}^{0}, \phi_{i}\right\}$ for three-point functions of generic vector bosons and

$$
A_{3}\left(\Phi_{i}, W_{j}^{+}, \hat{W}_{n}^{-, \mathrm{A}}\right)=0
$$


for three-point functions involving the reference leg. The selection rules for longitudinal vector bosons hold since the choice of spin axes ensures

$$
\epsilon\left(k_{j},+\right) \cdot r_{i}=0=\hat{\epsilon}^{\mathrm{A}}\left(k_{n},-\right) \cdot r_{i}
$$

for all legs $i$ and $j$. In analogy to the discussion for the Risager shift, it is seen that all possible combinations of three-point amplitudes contributing to the recursive construction of the four-point amplitude vanish,

$$
\begin{aligned}
& \sum_{s} A_{3}\left(\Phi_{1}, \hat{W}_{l}^{+, \mathrm{H}}, \hat{\Phi}_{\mathcal{F}}^{s}\right) A_{3}\left(\hat{\Phi}_{\mathcal{F}^{\prime}}^{-s}, \hat{W}_{m}^{+, \mathrm{H}}, \hat{W}_{n}^{-, \mathrm{A}}\right)=0 \\
& \sum_{s} A_{3}\left(\hat{W}_{l}^{+, \mathrm{H}}, \hat{W}_{m}^{+, \mathrm{H}}, \hat{\Phi}_{\mathcal{F}}^{s}\right) A_{3}\left(\hat{\Phi}_{\mathcal{F}^{\prime}}^{-s}, \Phi_{1}, \hat{W}_{n}^{-, \mathrm{A}}\right)=0 .
\end{aligned}
$$

The selection rules (6.3) for arbitrary multiplicities follow by induction using the recursion relation.

\subsection{Selection rules for amplitudes with a fermion pair}

Similar to the bosonic case, the selection rule (6.4) for a fermion pair and an arbitrary number of vector bosons with identical spin labels can be established by a three-line antiholomorphic Risager shift $\psi_{l}^{-, \mathrm{A}} W_{m}^{-, \mathrm{A}} W_{n}^{-, \mathrm{A}}$. In the recursion relation, contributions from internal boson and fermion lines must be taken into account,

$$
\begin{aligned}
& A\left(\bar{\psi}_{1}^{-}, \ldots, \psi_{l}^{-}, \ldots, W_{m}^{-}, \ldots, W_{n}^{-}\right) \\
& =\sum_{\mathcal{F}, s} A_{\mathcal{F}}\left(\bar{\psi}_{1}^{-}, \ldots \hat{\psi}_{l}^{-, \mathrm{A}} \ldots \hat{\Phi}_{\mathcal{F}}^{s}\right) \frac{\mathrm{i}}{K_{\mathcal{F}}^{2}-M_{\mathcal{F}}^{2}} A_{\mathcal{F}^{\prime}}\left(\hat{\Phi}_{\mathcal{F}^{\prime}}^{-s}, \ldots, \hat{W}_{m}^{-, \mathrm{A}}, \ldots \hat{W}_{n}^{-, \mathrm{A}} \ldots\right) \\
& \quad+\sum_{\mathcal{F}, s} A_{\mathcal{F}}\left(\bar{\psi}_{1}^{-}, \ldots \hat{W}_{m}^{-, \mathrm{A}} \ldots \hat{\psi}_{\mathcal{F}}^{s}\right) \frac{\mathrm{i}}{K_{\mathcal{F}}^{2}-M_{\mathcal{F}}^{2}} A_{\mathcal{F}^{\prime}}\left(\hat{\bar{\psi}}_{\mathcal{F}^{\prime}}^{-s}, \ldots, \hat{\psi}_{l}^{-, \mathrm{A}}, \ldots \hat{W}_{n}^{-, \mathrm{A}} \ldots\right)+\ldots,
\end{aligned}
$$

where again the dots indicate contributions with a different distribution of the shifted legs over the subamplitudes.

Selection rules for the three-point fermion amplitudes can be inferred from the contractions of Dirac spinors (2.23) with the polarization vectors (2.35),

$$
\notin\left(k_{i},-\right) u\left(k_{j},-\frac{1}{2}\right) \propto\left(\begin{array}{c}
-k_{i, \alpha}^{b} m_{j} \frac{\left[q_{i} q_{j}\right]}{\left[q_{j} k_{j}^{b}\right]} \\
q_{i, \dot{\alpha}}\left\langle k_{i}^{b} k_{j}^{b}\right\rangle
\end{array}\right) .
$$

This implies the selection rule for three-point functions for generic legs, ${ }^{10}$

$$
A_{3}\left(\psi_{i}^{-}, W_{j}^{-}, \psi_{k}^{-}\right)=0
$$

since the same anti-holomorphic reference spinors enter the left-handed polarization vectors and spinors of all external or internal particles, including the reference leg.

\footnotetext{
${ }^{10}$ For simplicity vector-like couplings are assumed. The presence of different left- and right-handed couplings does not change the results, while for purely chiral couplings additional selection rules can arise.
} 
In the recursive construction of the four-point amplitude using (6.18), the contributing products of three-point amplitudes all vanish for arbitrary spin states of the intermediate particle,

$$
\begin{aligned}
& \sum_{s} A_{3}\left(\bar{\psi}_{1}^{-}, \hat{\psi}_{l}^{-, \mathrm{A}}, \hat{\Phi}_{\mathcal{F}}^{s}\right) A_{3}\left(\hat{\Phi}_{\mathcal{F}^{\prime}}^{-s}, \hat{W}_{m}^{-, \mathrm{A}}, \hat{W}_{n}^{-, \mathrm{A}}\right)=0 \\
& \sum_{s} A_{3}\left(\bar{\psi}_{1}^{-}, \hat{W}_{m}^{-, \mathrm{A}}, \hat{\psi}_{\mathcal{F}}^{s}\right) A_{3}\left(\hat{\bar{\psi}}_{\mathcal{F}^{\prime}}^{-s}, \hat{\psi}_{l}^{-, \mathrm{A}}, \hat{W}_{n}^{-, \mathrm{A}}\right)=0 .
\end{aligned}
$$

Note that the selection rules for the bosonic vertices (6.9) ensure that the properties of the vertices involving fermions and scalars or longitudinal vector bosons are not required to show that the four-point amplitude vanishes. Due to the structure of the recursion relation (6.18), the selection rule (6.4) follows from induction.

The selection rules (6.5) and (6.6) can be derived using a mostly-holomorphic BCFWtype shift $\psi_{l}^{+, \mathrm{H}} W_{m}^{+, \mathrm{H}} W_{n}^{-, \mathrm{A}}$ or $W_{l}^{+, \mathrm{H}} W_{m}^{+, \mathrm{H}} \psi_{n}^{-, \mathrm{A}}$. In analogy to (6.20), the identities

$$
A_{3}\left(\psi_{i}^{+}, W_{j}^{+}, \psi_{k}^{+}\right)=0
$$

hold since the same holomorphic reference spinor enters all wave functions of particles with positive spin projection. For three-point amplitudes involving the reference particles, selection rules can be inferred from the contractions

$$
\notin\left(k_{i},+\right) \hat{u}^{\mathrm{A}}\left(k_{n},-\frac{1}{2}\right) \propto\left(\begin{array}{c}
-q_{i, \alpha} m_{n} \frac{\left[k_{i}^{\mathrm{b}} q_{n}\right]}{\left[q_{n} k_{n}^{\mathrm{b}}\right]} \\
k_{i, \dot{\alpha}}^{b}\left\langle q_{i} k_{n}^{b}\right\rangle
\end{array}\right), \quad \nexists^{\mathrm{A}}\left(k_{n},-\right) u\left(k_{j},+\frac{1}{2}\right) \propto\left(\begin{array}{c}
k_{n, \alpha}^{b}\left[q_{n} k_{j}^{\mathrm{b}}\right] \\
-q_{n, \dot{\alpha}} m_{j} \frac{\left\langle k_{n}^{\mathrm{b}} q_{j}\right\rangle}{\left\langle k_{j}^{\mathrm{b}} q_{j}\right\rangle}
\end{array}\right) .
$$

For the above choice of reference spinors, these imply the selection rules

$$
A_{3}\left(\bar{\psi}_{i}^{+}, W_{j}^{+}, \hat{\psi}_{n}^{-, \mathrm{A}}\right)=0, \quad A_{3}\left(\bar{\psi}_{i}^{+}, \psi_{k}^{+}, \hat{W}_{n}^{-, \mathrm{A}}\right)=0
$$

if a vector boson or fermion is used as reference particle.

The selection rule (6.5) is again derived inductively from the recursion relation, which has a similar form as (6.18). For the four-point amplitude this is seen since all products of three-point functions appearing in the recursive construction vanish,

$$
\begin{aligned}
& \sum_{\Phi, s} A_{3}\left(\bar{\psi}_{1}^{+}, \hat{\psi}_{l}^{+, \mathrm{H}}, \hat{\Phi}_{\mathcal{F}}^{s}\right) A_{3}\left(\hat{\Phi}_{\mathcal{F}^{\prime}}^{-s}, \hat{W}_{m}^{+, \mathrm{H}}, \hat{W}_{n}^{-, \mathrm{A}}\right)=0 \\
& \sum_{s} A_{3}\left(\bar{\psi}_{1}^{+}, \hat{W}_{m}^{+, \mathrm{H}}, \hat{\psi}_{\mathcal{F}}^{s}\right) A_{3}\left(\hat{\bar{\psi}}_{\mathcal{F}^{\prime}}^{-s}, \hat{\psi}_{l}^{+, \mathrm{H}}, \hat{W}_{n}^{-, \mathrm{A}}\right)=0 .
\end{aligned}
$$

The selection rule (6.6) is derived analogously, where the contributions in the four-point case are

$$
\begin{aligned}
& \sum_{\Phi, s} A_{3}\left(\hat{W}_{l}^{+, \mathrm{H}}, \hat{W}_{m}^{+, \mathrm{H}}, \hat{\Phi}_{\mathcal{F}}^{s}\right) A_{3}\left(\hat{\Phi}_{\mathcal{F}^{\prime}}^{-s}, \bar{\psi}_{1}^{+}, \hat{\psi}_{n}^{-, \mathrm{A}}\right)=0 \\
& \sum_{s} A_{3}\left(\bar{\psi}_{1}^{+}, \hat{W}_{m}^{+, \mathrm{H}}, \hat{\psi}_{\mathcal{F}}^{s}\right) A_{3}\left(\hat{\bar{\psi}}_{\mathcal{F}^{\prime}}^{-s}, \hat{W}_{l}^{+, \mathrm{H}}, \hat{\psi}_{n}^{-, \mathrm{A}}\right)=0 .
\end{aligned}
$$




\subsection{Transformation to a common spin axis}

The selection rules derived so far hold for a different spin axis (6.1) for legs $i \in\{1, \ldots, n-1\}$ compared to leg $n$. Little-group transformations can be used to transform to a convention where the same reference spinors are used for all particles. Taking the common reference spinors to be those of the reference particle $n$, the new reference spinors are given by

$$
q_{i, \alpha}^{\prime}=q_{n, \alpha}, \quad q_{i, \dot{\alpha}}^{\prime}=q_{i, \dot{\alpha}}=q_{n, \dot{\alpha}} .
$$

The expressions for the little-group rotation (B.3) imply that the elements of the transformation matrices for legs $i$ satisfy $R_{+-}=0$ and $R_{--}=R_{++}^{-1}$. For the comparison with the selection rules for a common spin axis [26], the explicit expressions of the transformations are not required, but rather the behaviour in the high-energy limit $E \gg m_{W}, m_{\psi}$. Using the scaling $\left.\left|k_{i}\right\rangle \sim \mid k_{i}\right] \sim \sqrt{E_{i}}$, the matrix elements behave in the high-energy limit as ${ }^{11}$

$$
R_{--} \sim 1, \quad R_{-+} \sim \frac{m_{i}}{E_{i}}, \quad R_{++} \sim 1 .
$$

Therefore the matrices implementing the transformation of the Dirac spinors (B.4) and vector-boson polarization vectors (B.5) are of upper triangular form, with the off-diagonal elements suppressed by powers of $m / E$,

$$
\left(\begin{array}{l}
u^{\prime}\left(+\frac{1}{2}\right) \\
u^{\prime}\left(-\frac{1}{2}\right)
\end{array}\right)=\left(\begin{array}{cc}
\mathcal{O}(1) & \mathcal{O}\left(\frac{m_{\psi}}{E}\right) \\
0 & \mathcal{O}(1)
\end{array}\right)\left(\begin{array}{l}
u\left(+\frac{1}{2}\right) \\
u\left(-\frac{1}{2}\right)
\end{array}\right),
$$

and

$$
\left(\begin{array}{c}
\epsilon^{\prime}(+) \\
\epsilon^{\prime}(0) \\
\epsilon^{\prime}(-)
\end{array}\right)=\left(\begin{array}{ccc}
\mathcal{O}(1) & \mathcal{O}\left(\frac{m_{W}}{E}\right) & \mathcal{O}\left(\left(\frac{m_{W}}{E}\right)^{2}\right) \\
0 & 1 & \mathcal{O}\left(\frac{m_{W}}{E}\right) \\
0 & 0 & \mathcal{O}(1)
\end{array}\right)\left(\begin{array}{c}
\epsilon(+) \\
\epsilon(0) \\
\epsilon(-)
\end{array}\right) .
$$

The resulting expressions for $\epsilon^{\prime}(-), \epsilon^{\prime}(0)$ and $u^{\prime}\left(-\frac{1}{2}\right)$ imply that the selection rules (6.2) and (6.4) for equal spin quantum numbers also hold exactly for a common spin axis. In contrast, the amplitudes (6.3), (6.5), and (6.6) do not vanish for a common spin axis but are suppressed by powers of $m / E$. For the amplitudes with only vector bosons, one finds

$$
\begin{aligned}
A^{\prime}\left(W_{1}^{+}, \ldots, W_{n}^{-}\right) \sim & \sum_{i, j}\left(\frac{m_{W_{i}} m_{W_{j}}}{E_{i} E_{j}}\right) A\left(W_{1}^{+}, \ldots W_{i}^{0}, \ldots, W_{j}^{0}, \ldots, W_{n}^{-}\right) \\
& +\sum_{i}\left(\frac{m_{W_{i}}}{E_{i}}\right)^{2} A\left(W_{1}^{+}, \ldots W_{i}^{-}, \ldots, W_{n}^{-}\right)+\ldots, \\
A^{\prime}\left(W_{1}^{0}, W_{2}^{+}, \ldots, W_{n}^{-}\right) \sim & \left(\frac{m_{W_{1}}}{E_{1}}\right) A\left(W_{1}^{-}, W_{2}^{+}, \ldots, W_{n}^{-}\right) \\
& +\sum_{i}\left(\frac{m_{W_{i}}}{E_{i}}\right) A\left(W_{1}^{0}, W_{2}^{+}, \ldots W_{i}^{0}, \ldots, W_{n}^{-}\right) \ldots
\end{aligned}
$$

where the dots indicate contributions that are suppressed by higher powers of $(m / E)$. The amplitudes with two negative spin labels on the right-hand side of these identities are

\footnotetext{
${ }^{11}$ These relations hold independent of the scaling of the reference spinors in the high-energy limit.
} 
non-vanishing in the massless limit, where they are just the MHV amplitudes. The same holds for the amplitudes $A\left(\phi_{1}, W_{2}^{+}, \ldots \phi_{i}, \ldots, W_{n}^{-}\right)$, which arise from the high-energy limit of amplitudes with two longitudinal vector bosons. Therefore, all suppression factors are explicit in the above relations. These results agree with table 1 in [26].

For amplitudes with a scalar one obtains

$$
A^{\prime}\left(\phi_{1}, W_{2}^{+}, \ldots, W_{n}^{-}\right) \sim \sum_{i}\left(\frac{m_{W_{i}}}{E_{i}}\right) A\left(\phi_{1}, W_{2}^{+}, \ldots W_{i}^{0}, \ldots, W_{n}^{-}\right)+\ldots
$$

In the high-energy limit, the amplitude on the right-hand side again tend to MHV-type amplitudes with two scalars.

For the fermionic amplitudes (6.5) the little-group transformation generates two types of contributions with a $(m / E)^{1}$ suppression factor,

$$
\begin{aligned}
A^{\prime}\left(\bar{\psi}_{1}^{+}, \psi_{2}^{+}, W_{3}^{+}, \ldots W_{n}^{-}\right) \sim & \sum_{i=1,2}\left(\frac{m_{\psi_{i}}}{E_{i}}\right) A\left(\bar{\psi}_{1}^{+}, \psi_{i}^{-}, W_{3}^{+}, \ldots, W_{n}^{-}\right) \\
& +\sum_{i}\left(\frac{m_{W_{i}}}{E_{i}}\right) A\left(\bar{\psi}_{1}^{+}, \psi_{2}^{+}, W_{3}^{+}, \ldots, W_{i}^{0}, \ldots W_{n}^{-}\right)+\ldots
\end{aligned}
$$

Here the amplitudes in the first term on the right-hand side are of MHV type and therefore un-suppressed in the high-energy limit. Also the amplitudes in the second line are non-vanishing in the high-energy limit, as can be seen by applying the Goldstone-boson equivalence theorem and using the helicity structure of the Yukawa coupling. ${ }^{12}$ Transforming the selection rule (6.6) to a common spin axis yields

$$
\begin{aligned}
A^{\prime}\left(\bar{\psi}_{1}^{+}, W_{2}^{+}, \ldots \psi_{n}^{-}\right) \sim & \left(\frac{m_{\psi_{1}}}{E_{1}}\right) A\left(\bar{\psi}_{1}^{-}, W_{2}^{+}, \ldots \psi_{n}^{-}\right) \\
& +\sum_{i}\left(\frac{m_{W_{i}}}{E_{i}}\right) A\left(\bar{\psi}_{1}^{+}, W_{2}^{+}, \ldots W_{i}^{0}, \ldots \psi_{n}^{-}\right) \\
& +\sum_{i}\left(\frac{m_{W_{i}}}{E_{i}}\right)^{2} A\left(\bar{\psi}_{1}^{+}, W_{2}^{+}, \ldots W_{i}^{-}, \ldots \psi_{n}^{-}\right)+\ldots
\end{aligned}
$$

Those amplitudes on the right-hand side that are multiplied by a factor of $(m / E)^{1}$ are not of MHV type and vanish in the massless limit by helicity conservation, in case of the amplitudes involving longitudinal vector bosons after application of the Goldstone-boson equivalence theorem. Therefore these amplitudes are themselves suppressed by a factor of $m_{\psi} / E$ relative to the MHV-type amplitudes in the last line, so that all terms on the right-hand side are effectively of order $\sim m_{i} m_{j} / E^{2}$ for different combinations of fermion or vector-boson masses. This is consistent with all-multiplicity results for amplitudes for massive quarks and massless vector bosons [21, 43].

The above observations shed some new light on the results of [26]: amplitudes that are mass-suppressed for common spin axes may vanish exactly for some other choice of spin

\footnotetext{
${ }^{12}$ Here the high-energy limit is interpreted as the limit $(v / E) \rightarrow 0$ with the scalar vacuum expectation value $v$. If the limit $m_{\psi} \rightarrow 0$ is instead implemented by sending the fermion Yukawa couplings to zero, these amplitudes vanish.
} 
axes. In fact, the scaling (6.31) is not limited to the particular choice (6.1) of the initial spin axes but holds generically in the high-energy limit. However, in general $R_{+-}$is nonvanishing but of order $m / E$, so the sets of amplitudes (6.2) and (6.4) are expected to be mass-suppressed for general choices of the spin axes. Therefore, amplitudes that vanish for one choice of spin axes are mass-suppressed for generic choices. These results are consistent with the vanishing of these amplitudes in the massless limit, but it is interesting to see them emerge from the little-group transformations, which also allow to obtain the power of the suppression factors.

\section{Summary and conclusions}

We have performed a comprehensive study of complex deformations of Born amplitudes in spontaneously broken gauge theories and analysed the behaviour for large values of the deformation parameter $z$, building on previous studies $[4,5,23]$. Based on these results we have identified the minimal shifts necessary to obtain valid on-shell recursion relations for amplitudes with a given particle content and spin quantum numbers. Spontaneously broken gauge invariance has been shown to improve the large- $z$ behaviour through the use of Ward-identities. Since an on-shell construction of three-point and four-point amplitudes can be performed using little-group invariance and factorization arguments [11], we have focused on amplitudes with five or more legs. We find that two-line or three-line shifts are sufficient for all such amplitudes involving at least two transverse gauge bosons, amplitudes with fermions and vector bosons, and for purely fermionic amplitudes with six or more legs. Furthermore, all amplitudes with multiple SM-like Higgs bosons and fermions or at least one transverse vector boson are three-line constructible. For all remaining amplitudes fourline shifts are sufficient, with the exception of pure scalar amplitudes where five-line shifts are required. As application, we have shown how selection rules for massive multi-boson amplitudes follow from on-shell recursion relations and have explored the role of the choice of spin axes using little-group transformations.

Our results for the minimal shifts are in overall agreement with those for unbroken gauge theories with the same matter content [5], up to the results for longitudinal vector bosons, which only arise in the massive case. This is intuitively expected from the analogy of the large- $z$ behaviour and the high-energy limit. However, as demonstrated by some examples, amplitudes with massive fermions and vector bosons may show worse large- $z$ behaviour under extended BCFW-type shifts than massless amplitudes due to the dependence on the spin axis or the appearance of contributions that are forbidden in the massless case by the helicity structure.

We have presented little-group covariant expressions for shifted momenta and wave functions but made a particular choice of spin axes to find valid shifts for all spin configurations, so our final prescriptions for the shifts are not manifestly little-group invariant. The possibility to use the covariant form for mixed shifts of massive and massless particles [30] deserves further study. The question remains if a "spin blind" recursive construction is possible, which does not require to specify the spin axes of massive particles. It would be 
interesting to explore a possible extension of a definition of shifts for generic polarizations of massless vector bosons [44] to the massive case.

While we have limited the discussion to renormalizable gauge theories with spin $s \leq 1$, the methods used for the estimate of the large- $z$ behaviour of massive amplitudes could be extended towards effective field theories or to higher-spin particles. In the former case, on-shell recursion relations for non-linearly realised effective theories of massless particles have been studied [6] while the application to the extension of the Standard Model by higher-dimensional operators has been initiated recently [30]. An initial application of on-shell recursion relations to amplitudes with massive spin-two particles was given in [45].

\section{Acknowledgments}

The work of RF was supported by the DFG project "Precise predictions for vector-boson scattering at hadron colliders" (project no. 322921231) while CS acknowledges support by the Heisenberg Programme of the DFG.

\section{A Conventions}

The spinor conventions used in this paper follow [23]. The sigma matrices are defined as $\sigma_{\alpha \dot{\beta}}^{\mu}=(1,-\vec{\sigma}), \bar{\sigma}^{\mu \dot{\alpha} \beta}=(1, \vec{\sigma})$, where $\vec{\sigma}=\left(\sigma_{x}, \sigma_{y}, \sigma_{z}\right)$ are the Pauli matrices. Four-vectors $x^{\mu}$ are mapped to bi-spinors according to

$$
x_{\alpha \dot{\alpha}}=x_{\mu} \sigma_{\alpha \dot{\alpha}}^{\mu}, \quad x^{\dot{\alpha} \alpha}=x_{\mu} \bar{\sigma}^{\mu ; \dot{\alpha} \alpha} .
$$

The conventions for the two-dimensional antisymmetric tensor are given by

$$
\varepsilon^{\alpha \beta}=\varepsilon^{\dot{\alpha} \dot{\beta}}=\varepsilon_{\alpha \beta}=\varepsilon_{\dot{\alpha} \dot{\beta}}=\left(\begin{array}{cc}
0 & 1 \\
-1 & 0
\end{array}\right) .
$$

Indices of two-component Weyl spinors are raised and lowered as follows:

$$
k^{\alpha}=\varepsilon^{\alpha \beta} k_{\beta}, \quad k^{\dot{\alpha}}=\varepsilon^{\dot{\alpha} \dot{\beta}} k_{\dot{\beta}}, \quad k_{\dot{\beta}}=k^{\dot{\alpha}} \varepsilon_{\dot{\alpha} \dot{\beta}}, \quad k_{\beta}=k^{\alpha} \varepsilon_{\alpha \beta} .
$$

In the bra-ket notation spinor products are denoted as

$$
\langle p q\rangle=p^{\alpha} q_{\alpha}, \quad[q p]=q_{\dot{\alpha}} p^{\dot{\alpha}},
$$

while contractions of spinors with "slashed" momenta are given by

$$
\langle p|\not k| q]=p^{\alpha} k_{\alpha \dot{\beta}} q^{\dot{\alpha}}, \quad[p|\not k| q\rangle=p_{\dot{\alpha}} k^{\dot{\alpha} \beta} q_{\beta} .
$$

Explicit solutions for the Weyl spinors associated to a light-like momentum can be written as

$$
k_{\alpha}=\frac{e^{\frac{i}{2}(\chi-\phi)}}{\sqrt{k_{0}+k_{3}}}\left(\begin{array}{c}
-k_{1}+\mathrm{i} k_{2} \\
k_{0}+k_{3}
\end{array}\right), \quad k^{\dot{\alpha}}=\frac{e^{-\frac{i}{2}(\chi+\phi)}}{\sqrt{k_{0}+k_{3}}}\left(\begin{array}{c}
k_{0}+k_{3} \\
k_{1}+\mathrm{i} k_{2}
\end{array}\right) \text {, }
$$


where $\phi$ is defined by $k_{0}+k_{3}=\left|k_{0}+k_{3}\right| e^{\mathrm{i} \phi}$. In the convention of [23], the arbitrary phase $\chi$ is set to zero. With this convention, the complex conjugate spinors for real momenta satisfy

$$
\left(k_{\alpha}\right)^{*}=\operatorname{sgn}\left(k^{+}\right) k_{\dot{\alpha}}, \quad\left(k^{\dot{\alpha}}\right)^{*}=\operatorname{sgn}\left(k^{+}\right) k^{\alpha},
$$

with $k^{+}=k^{0}+k^{3}$. This implies the relations for the spinor products

$$
\langle k q\rangle^{*}=[q k], \quad[q k]^{*}=\langle k q\rangle
$$

if $k^{+}, q^{+}>0$. The relations for the Weyl spinors for crossed momenta, $p=-k$ are given by

$$
p_{\alpha}=\mathrm{i} \operatorname{sgn}\left(k^{+}\right) k_{\alpha}, \quad p^{\dot{\alpha}}=\mathrm{i} \operatorname{sgn}\left(k^{+}\right) k^{\dot{\alpha}}, \quad p^{\alpha}=\mathrm{i} \operatorname{sgn}\left(k^{+}\right) k^{\alpha}, \quad p_{\dot{\alpha}}=\mathrm{i} \operatorname{sgn}\left(k^{+}\right) k_{\dot{\alpha}} .
$$

\section{B Little-group transformations}

Since we have shown the validity of on-shell recursion relations for a particular choice of the spin axes made in section 5.1, it is necessary to perform little-group rotations to obtain results in general frames. According to the dictionary (2.9), a transformation of reference spinors $\left\{q_{\alpha}, q_{\dot{\alpha}}\right\} \rightarrow\left\{q_{\alpha}^{\prime}, q_{\dot{\alpha}}^{\prime}\right\}$ can be obtained by finding a little-group transformation that takes $k_{\alpha}^{2} \rightarrow k_{\alpha}^{\prime 2}$ and $k_{\dot{\alpha}, 2} \rightarrow k_{\dot{\alpha}, 2}^{\prime}$ (i.e. $\left.k_{\dot{\alpha}}^{1} \rightarrow k_{\dot{\alpha}}^{\prime 1}\right)$. This is achieved by the transformation with components

$$
R_{I}^{1}=\frac{1}{m}\left[k^{\prime 1} k_{I}\right], \quad \quad R_{I}^{2}=-\frac{1}{m}\left\langle k^{\prime 2} k_{I}\right\rangle .
$$

It can be verified that the condition

$$
\varepsilon^{K L} R_{K}^{I} R_{L}^{J}=\varepsilon^{I J}
$$

is satisfied due to the Dirac equation (2.5) and the normalization condition (2.7). Note that both the primed and unprimed spinors correspond to the same four-momentum and satisfy the corresponding Dirac equation. Making the relation to the spin-axis notation explicit, the components of $R$ are given by

$$
\begin{array}{ll}
R^{1}{ }_{1}=\frac{\left\langle k^{\prime b} q\right\rangle}{\left\langle k^{\mathrm{b}} q\right\rangle}=\frac{\left[q^{\prime}|\not k| q\right\rangle}{\left[q^{\prime} k^{\prime b}\right]\left\langle k^{b} q\right\rangle} \equiv R_{--}, & R^{1}{ }_{2}=-\frac{m\left[q^{\prime} q\right]}{\left[q^{\prime} k^{\prime b}\right]\left[k^{b} q\right]} \equiv R_{+-}, \\
R^{2}{ }_{1}=-\frac{m\left\langle q^{\prime} q\right\rangle}{\left\langle q^{\prime} k^{\prime b}\right\rangle\left\langle k^{b} q\right\rangle} \equiv R_{-+}, & R^{2}{ }_{2}=\frac{\left[k^{\prime b} q\right]}{\left[k^{b} q\right]}=\frac{\left\langle q^{\prime}|\not k| q\right]}{\left\langle q^{\prime} k^{\prime b}\right\rangle\left[k^{b} q\right]} \equiv R_{++},
\end{array}
$$

where $k^{\prime b}$ refers to the light-cone projection of $k$ with respect to the reference vector $q^{\prime}$.

The transformation of Dirac spinors under little group transformations (2.22) implies the transformation of the spinors in the spin basis (2.27) with the matrix

$$
\mathcal{R}^{\left(\frac{1}{2}\right)}=\left(\begin{array}{ll}
R_{++} & R_{-+} \\
R_{+-} & R_{--}
\end{array}\right), \quad \quad \mathcal{R}^{\left(\frac{1}{2}\right)-1}=\left(\begin{array}{cc}
R_{--} & -R_{+-} \\
-R_{-+} & R_{++}
\end{array}\right) .
$$

These results agree with those in [23] with the identifications $R_{++}=c_{11}, R_{--}=c_{22}$, $R_{-+}=-c_{12}$, and $R_{+-}=-c_{21}$. 
The matrix representation of the little-group transformation of the polarization vectors (2.39) is found to be

$$
\mathcal{R}^{(1)}=\left(\begin{array}{ccc}
R_{++}^{2} & -\sqrt{2} R_{-+} R_{++} & -R_{-+}^{2} \\
\sqrt{2} R_{++} R_{+-} & R_{++} R_{--}+R_{-+} R_{+-} & \sqrt{2} R_{--} R_{-+} \\
-R_{+-}^{2} & \sqrt{2} R_{+-} R_{--} & R_{--}^{2}
\end{array}\right) .
$$

It satisfies $\operatorname{det} \mathcal{R}=1$ and respects the orthonormalization conditions of the polarization vectors,

$$
\mathcal{R}^{(1)} \gamma \mathcal{R}^{(1) T}=\gamma \quad \text { with } \quad \gamma_{s s^{\prime}}=\epsilon(s) \cdot \epsilon\left(s^{\prime}\right)=-\delta_{s,-s^{\prime}}
$$

\section{C $\quad Q_{\mathcal{F}}^{2} \neq 0$ shifts}

In an attempt to improve the large- $z$ behaviour, one may consider giving up the requirement of light-like shifts for internal lines (3.5) so that propagator denominators scale like $z^{2}$ instead of $z^{1}$. The form of the recursion relation valid for this case can be found in [5]. Since the shift vectors $\delta k_{i}$ are always light-like, momentum conservation implies that internal lines are necessarily light-like for two-line or three-line shifts. Shifts with $Q_{\mathcal{F}}^{2} \neq 0$ are thus obtained for at least four shifted lines and are only constrained by the condition of momentum conservation (3.34). Note that all shift spinors $\eta_{i}$ should be chosen differently since otherwise the internal shifts $Q_{\mathcal{F}, \alpha \dot{\alpha}}$ factorize into two Weyl spinors for some factorization channels and the corresponding propagator denominators "accidentally" scale like $z$. However, keeping all $\eta_{i}$ different implies that spinor products of shifted spinors can scale like $z^{2}$ so that the advantage of an improved large- $z$ scaling of the propagators is partially compensated by a worse behaviour of the numerator.

A bound for the large- $z$ behaviour for generic $Q_{\mathcal{F}}^{2} \neq 0$ shifts can be obtained using the reasoning of section 4.3. Despite choosing all $\eta_{i}$ differently, contributions with $Q_{\mathcal{F}}^{2}=0$ can appear due to background insertions into external shifted legs. The large- $z$ scaling of the propagator denominators $\gamma_{D}$ is therefore constrained by the mass-dimension of the denominators according to [5]

$$
\frac{1}{2}\left[D_{h, b}\right] \leq \gamma_{D} \leq\left[D_{h, b}\right]
$$

instead of (4.18). The value of $\left[D_{h, b}\right]-\gamma_{D}$ is given by the number of propagators with $Q_{\mathcal{F}}^{2}=0$. A conservative upper bound is obtained by assuming all background insertions couple through cubic vertices and lead to a propagator with light-like shift, so that

$$
\left[D_{h, b}\right]-\gamma_{D} \leq b .
$$

Instead of (4.24), the bound on the large- $z$ scaling of the amplitude becomes

$$
\begin{aligned}
\gamma & \leq 4-(h+b)-[g]-\frac{b_{\psi}}{2}+\left[D_{h, b}\right]-\gamma_{D}-\sum_{\mathcal{H}} s_{i}+\sum_{\mathcal{A}} s_{j} \\
& \leq 4-h-\min [g]-\sum_{\mathcal{H}} s_{i}+\sum_{\mathcal{A}} s_{j} .
\end{aligned}
$$


Therefore, all amplitudes in renormalizable theories are constructible from five-line shifts with $Q_{\mathcal{F}}^{2} \neq 0$ [5]. Since this offers no advantage over Risager-type shifts but leads to a more complicated recursion relation, we do not consider such shifts any further.

Open Access. This article is distributed under the terms of the Creative Commons Attribution License (CC-BY 4.0), which permits any use, distribution and reproduction in any medium, provided the original author(s) and source are credited.

\section{References}

[1] R. Britto, F. Cachazo and B. Feng, New recursion relations for tree amplitudes of gluons, Nucl. Phys. B 715 (2005) 499 [hep-th/0412308] [INSPIRE].

[2] R. Britto, F. Cachazo, B. Feng and E. Witten, Direct proof of tree-level recursion relation in Yang-Mills theory, Phys. Rev. Lett. 94 (2005) 181602 [hep-th/0501052] [INSPIRE].

[3] P. Benincasa and F. Cachazo, Consistency Conditions on the S-matrix of Massless Particles, arXiv: 0705.4305 [INSPIRE].

[4] T. Cohen, H. Elvang and M. Kiermaier, On-shell constructibility of tree amplitudes in general field theories, JHEP 04 (2011) 053 [arXiv: 1010.0257] [INSPIRE].

[5] C. Cheung, C.-H. Shen and J. Trnka, Simple Recursion Relations for General Field Theories, JHEP 06 (2015) 118 [arXiv: 1502.05057] [INSPIRE].

[6] C. Cheung, K. Kampf, J. Novotny, C.-H. Shen and J. Trnka, On-Shell Recursion Relations for Effective Field Theories, Phys. Rev. Lett. 116 (2016) 041601 [arXiv:1509.03309] [INSPIRE].

[7] N. Craig, H. Elvang, M. Kiermaier and T. Slatyer, Massive amplitudes on the Coulomb branch of $\mathcal{N}=4$ SYM, JHEP 12 (2011) 097 [arXiv:1104.2050] [INSPIRE].

[8] R.H. Boels and C. Schwinn, On-shell supersymmetry for massive multiplets, Phys. Rev. D 84 (2011) 065006 [arXiv:1104.2280] [INSPIRE].

[9] G. Chen and K.G. Savvidy, Spinor formalism for massive fields with half-integral spin, Eur. Phys. J. C 72 (2012) 1952 [arXiv:1105.3851] [INSPIRE].

[10] E. Conde and A. Marzolla, Lorentz Constraints on Massive Three-Point Amplitudes, JHEP 09 (2016) 041 [arXiv: 1601.08113] [INSPIRE].

[11] N. Arkani-Hamed, T.-C. Huang and Y.-t. Huang, Scattering Amplitudes For All Masses and Spins, arXiv:1709.04891 [INSPIRE].

[12] J.M. Cornwall, D.N. Levin and G. Tiktopoulos, Uniqueness of spontaneously broken gauge theories, Phys. Rev. Lett. 30 (1973) 1268 [Erratum ibid. 31 (1973) 572] [INSPIRE].

[13] J.M. Cornwall, D.N. Levin and G. Tiktopoulos, Derivation of Gauge Invariance from High-Energy Unitarity Bounds on the s Matrix, Phys. Rev. D 10 (1974) 1145 [Erratum ibid. D 11 (1975) 972] [INSPIRE].

[14] C.H. Llewellyn Smith, High-Energy Behavior and Gauge Symmetry, Phys. Lett. 46B (1973) 233 [INSPIRE].

[15] N. Christensen and B. Field, Constructive standard model, Phys. Rev. D 98 (2018) 016014 [arXiv: 1802.00448] [INSPIRE]. 
[16] N. Christensen, B. Field, A. Moore and S. Pinto, 2-, 3- and 4-Body Decays in the Constructive Standard Model, arXiv:1909.09164 [INSPIRE].

[17] G. Durieux, T. Kitahara, Y. Shadmi and Y. Weiss, The electroweak effective field theory from on-shell amplitudes, JHEP 01 (2020) 119 [arXiv:1909.10551] [INSPIRE].

[18] S.D. Badger, E.W.N. Glover, V.V. Khoze and P. Svrček, Recursion relations for gauge theory amplitudes with massive particles, JHEP 07 (2005) 025 [hep-th/0504159] [INSPIRE].

[19] S.D. Badger, E.W.N. Glover and V.V. Khoze, Recursion relations for gauge theory amplitudes with massive vector bosons and fermions, JHEP 01 (2006) 066 [hep-th/0507161] [INSPIRE].

[20] D. Forde and D.A. Kosower, All-multiplicity amplitudes with massive scalars, Phys. Rev. D 73 (2006) 065007 [hep-th/0507292] [INSPIRE].

[21] P. Ferrario, G. Rodrigo and P. Talavera, Compact multigluonic scattering amplitudes with heavy scalars and fermions, Phys. Rev. Lett. 96 (2006) 182001 [hep-th/0602043] [INSPIRE].

[22] K.J. Ozeren and W.J. Stirling, Scattering amplitudes with massive fermions using BCFW recursion, Eur. Phys. J. C 48 (2006) 159 [hep-ph/0603071] [INSPIRE].

[23] C. Schwinn and S. Weinzierl, On-shell recursion relations for all Born QCD amplitudes, JHEP 04 (2007) 072 [hep-ph/0703021] [INSPIRE].

[24] N. Arkani-Hamed and J. Kaplan, On Tree Amplitudes in Gauge Theory and Gravity, JHEP 04 (2008) 076 [arXiv:0801.2385] [INSPIRE].

[25] K. Risager, A direct proof of the CSW rules, JHEP 12 (2005) 003 [hep-th/0508206] [INSPIRE].

[26] F. Coradeschi and P. Lodone, Selection rules for helicity amplitudes in massive gauge theories, Phys. Rev. D 87 (2013) 074026 [arXiv:1211.1880] [InSPIRE].

[27] R. Kleiss and W.J. Stirling, Spinor Techniques for Calculating $p \bar{p} \rightarrow W^{ \pm} / Z^{0}+$ Jets, Nucl. Phys. B 262 (1985) 235 [INSPIRE].

[28] S. Dittmaier, Weyl-van der Waerden formalism for helicity amplitudes of massive particles, Phys. Rev. D 59 (1998) 016007 [hep-ph/9805445] [INSPIRE].

[29] A. Ochirov, Helicity amplitudes for QCD with massive quarks, JHEP 04 (2018) 089 [arXiv: 1802.06730] [INSPIRE].

[30] R. Aoude and C.S. Machado, The Rise of SMEFT On-shell Amplitudes, JHEP 12 (2019) 058 [arXiv: 1905.11433] [INSPIRE].

[31] G. Chalmers and W. Siegel, Simplifying algebra in Feynman graphs. 3. Massive vectors, Phys. Rev. D 63 (2001) 125027 [hep-th/0101025] [InSPIRE].

[32] A. Herderschee, S. Koren and T. Trott, Constructing $\mathcal{N}=4$ Coulomb branch superamplitudes, JHEP 08 (2019) 107 [arXiv: 1902.07205] [INSPIRE].

[33] C. Schwinn and S. Weinzierl, Scalar diagrammatic rules for Born amplitudes in QCD, JHEP 05 (2005) 006 [hep-th/0503015] [INSPIRE].

[34] B.W. Lee, C. Quigg and H.B. Thacker, Weak Interactions at Very High-Energies: The Role of the Higgs Boson Mass, Phys. Rev. D 16 (1977) 1519 [INSPIRE].

[35] M.S. Chanowitz and M.K. Gaillard, The TeV Physics of Strongly Interacting W's and Z's, Nucl. Phys. B 261 (1985) 379 [INSPIRE]. 
[36] R.H. Boels, No triangles on the moduli space of maximally supersymmetric gauge theory, JHEP 05 (2010) 046 [arXiv: 1003.2989] [INSPIRE].

[37] A. Azatov, R. Contino, C.S. Machado and F. Riva, Helicity selection rules and noninterference for BSM amplitudes, Phys. Rev. D 95 (2017) 065014 [arXiv:1607.05236] [INSPIRE].

[38] Y. Shadmi and Y. Weiss, Effective Field Theory Amplitudes the On-Shell Way: Scalar and Vector Couplings to Gluons, JHEP 02 (2019) 165 [arXiv:1809.09644] [INSPIRE].

[39] T. Ma, J. Shu and M.-L. Xiao, Standard Model Effective Field Theory from On-shell Amplitudes, arXiv:1902.06752 [INSPIRE].

[40] C. Cheung, On-Shell Recursion Relations for Generic Theories, JHEP 03 (2010) 098 [arXiv: 0808.0504] [INSPIRE].

[41] M.L. Mangano and S.J. Parke, Multiparton amplitudes in gauge theories, Phys. Rept. 200 (1991) 301 [hep-th/0509223] [INSPIRE].

[42] L.J. Dixon, Calculating scattering amplitudes efficiently, in QCD and beyond. Proceedings, Theoretical Advanced Study Institute in Elementary Particle Physics, TASI-95, Boulder, U.S.A., June 4-30, 1995, pp. 539-584, 1996, hep-ph/9601359 [INSPIRE].

[43] C. Schwinn and S. Weinzierl, SUSY ward identities for multi-gluon helicity amplitudes with massive quarks, JHEP 03 (2006) 030 [hep-th/0602012] [INSPIRE].

[44] L. Rodina, Uniqueness from locality and BCFW shifts, JHEP 09 (2019) 078 [arXiv: 1612.03885] [INSPIRE].

[45] N. Moynihan and J. Murugan, Comments on scattering in massive gravity, vDVZ and BCFW, Class. Quant. Grav. 35 (2018) 155005 [arXiv:1711.03956] [INSPIRE]. 